\begin{tabular}{|c|c|c|}
\hline & DJS Vol. 38 (2017) 81-93 \\
\hline 1969 & $\begin{array}{c}\text { Delta Journal of Science } \\
\text { Available online at } \\
\text { https://djs.journals.ekb.eg/ }\end{array}$ \\
\hline Research Article & MATHEMATICS \\
\hline
\end{tabular}

\title{
Multi-Item Probabilistic Buffer Stock Inventory Model with Zero Lead- time and Varying Holding Cost under a Restriction
}

\author{
Ass. Prof. Dr. : Fergany.H.A. ${ }^{\text {, }}$ Mob:+201223382806, E-mail: halafergany@Yahoo.com \\ Ass. Lecturer: Hollah.O.M.'.* , Mob: +201211152924, E-mail: eng_Hollah@Yahoo.com \\ ${ }^{a}$ Department of Mathematics, Faculty of Science, Tanta University, Egypt \\ ${ }^{b}$ Department of Mathematics, High Institute for Computers, Information \& Management, Tanta, Egypt
}

\begin{abstract}
This paper developed a probabilistic periodic review model with a constraint which on the storage space for crisp and fuzzy environment using geometric programming approach with the following characteristics: Demand is instantaneous and its distribution is continuous. The costs are considered item cost, order cost and holding cost. The expected total cost will be based on the cost per cycle rather than cost per period. Then a Numerical example is provided.
\end{abstract}

Keywords: Probabilistic inventory system; Geometric programming; Triangular fuzzy number; Periodic review model;

1. Introduction: In general the classical inventory problems are designed by considering that the cost of an item is constant. But in practical situation, for the owner of the store, the holding cost is varying, where the holding cost of an item inversely relates to the length of the cycle.

Abou - El-Ata and Kotb (1997) introduced multi-item deterministic EOQ inventory model with varying holding cost under two restrictions using geometric programming approach. Kotb and Fergany (2011) introduced multi-item EOQ model with both demand-dependent unit cost and varying leading time via Geometric Programming. A considerable amount of inventory control literature concerns the computation of $\left(Q_{m}, N\right)$ policies under
Scarf's assumptions as in Feng and Xiao (2000). Abuo-El-Ata et al. (2003)

illustrated Probabilistic multi-item inventory model with varying order cost under two restrictions using geometric programming approach. Fergany (2005) developed a Periodic review probabilistic multi-item inventory system with zero lead time under constraints and varying ordering cost using Lagrange multiplier technique. Chiang (2008) introduced a Periodic review inventory models with stochastic supplier's visit intervals with constant units costs. In this regard, $\left(Q_{m}, N\right)$ policy in which the replenishment is set every $\mathrm{N}$ periods to raise the inventory position to the order-up-to-level $Q_{m}$ - provides an effective means of dampening the planning instability and coping 
with demand uncertainty. Furthermore, as pointed out by Silver et al. (1998), $\left(Q_{m}, N\right)$ is particularly appealing when items are ordered from the same supplier or require resource sharing.

In such a case all items in a coordinated group can be given the same replenishment period. Periodic review also allows a reasonable prediction of the level of the workload on the staff involved and is particularly suitable for advanced planning environments. For these reasons, as stated by Silver et al. (1998), $\left(Q_{m}, N\right)$ is a popular inventory policy. An important class of stochastic production/inventory control problems assumes a non-stationary demand process. Instead of employing a penalty cost scheme as in Tarim and Kingsman (2006) for $\left(Q_{m}{ }^{n}, N^{n}\right)$ under a penalty cost scheme. The optimal solution to the problem is the $\left(Q_{m}, N\right)$ policy with different values for each period in the time varying demand situation. Other notable works on non-stationary stochastic demand adopt $\left(Q_{m}, N\right)$ or base-stock policies and are due to Iida (1999), Sobel and Zhang (2001). InIida (1999), the periodic review dynamic inventory problem is considered and it is shown that near myopic policies are sufficiently close to optimal decisions for the infinite horizon inventory problem. In Sobel and Zhang (2001), it is assumed that demand arrives simultaneously from a deterministic source and a random source, and proven that a modified $\left(Q_{m}, N\right)$ policy is optimal under general conditions. Shashank et al. (2012) presented a periodic tabular policy for scheduling of a single stage productioninventory system. Tarim and Smith (2008) explained Constraint programming for computing non-stationary $\left(Q_{m}, N\right)$ inventory policies.

The cost parameters in real inventory systems and other parameters such as price, marketing and service elasticity to demand are imprecise and uncertain in nature. So, the notion of fuzziness can be applied to cope with this uncertainty. Since the proposed model is in a fuzzy environment, a fuzzy decision should be made to meet the decision criteria, and the results should be fuzzy. Fuzzy sets introduced by many researchers as a mathematical way of representing impreciseness or vagueness in everyday life. Samadi et al. (2013) illustrated Fuzzy pricing, marketing and service planning in a fuzzy inventory model: A geometric programming approach. Sadjadi et al. (2010) introduced Fuzzy pricing and marketing planning model: a possibilistic geometric programming approach. Liu (2007) used the geometric programming with fuzzy parameters in engineering optimization. Islam (2008) explained multi-objective marketing planning inventory model: A geometric programming approach. Islam and Roy (2007) developed Fuzzy multi-item economic production quantity model under space constraint: A geometric programming approach. Boydetet al. (2007) presenteda tutorial on geometric programming.

This paper develop multi-item probabilistic buffer stock inventory model with zero lead-time and varying holding cost under a restriction for crisp and fuzzy environment using geometric programming approach. The holding cost is considered as varying of the time of review. Furthermore, Demand is instantaneous in that all demand occurs at the beginning of the cycle, decreasing the stock level by that quantity. Our aim is to find the optimal maximum inventory level, the optimal time of review which minimizes the expected total cost.The model is illustrated witha numerical example for evaluation and validation of the results of model.The system is represented graphically in Figure (1). 


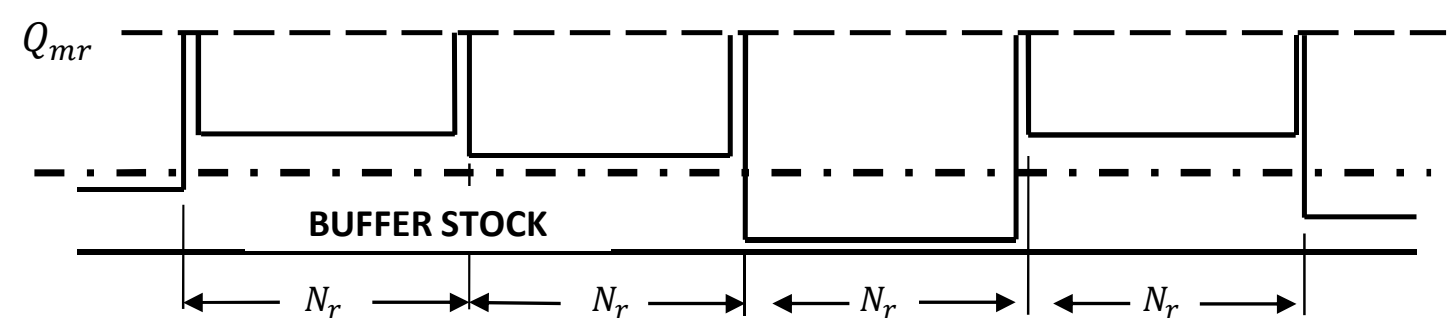

Figure (1): Inventory process with instantaneous demand

\section{Notations and assumptions}

Parameters for the $r^{\text {th }}(r=1,2, \ldots, n)$ item are

$C_{p r} \quad$ the item cost for $r^{\text {th }}$ item.

$C_{h r} \quad$ the holding cost per unit item per unit time.

$N_{r} \quad$ the review time (cycle) (decision variable).

$\beta_{r} \quad$ a constant real number selected toprovide the best fit of estimated

expected cost function such that $0<\beta_{r}<1$

$C_{h r}\left(N_{r}\right)$ the varying holding cost for the $r^{t h}$ per cycle $=C_{h r} N_{r}{ }^{-\beta_{r}}$

$C_{o r} \quad$ the order cost per unit item per cycle.

$S_{r} \quad$ the required warehouse space per unit of the $r^{\text {th }}$ item.

$Q_{m r} \quad$ maximum inventory level for the $r^{\text {th }}$ item, at timet $=0($ decision variable).

$x_{r}, \quad$ The random variable representing the demand of the $r^{\text {th }}$ item during the cycle $N_{r}$.

$x_{u r} \quad$ the maximum demand for the $r^{\text {th }}$ item during a cycle.

$E(T C)$ expected total average cost function $E\left(T C\left(Q_{m r}, N_{r}\right)\right)$ for the $r^{t h}$ item

$\tilde{C}_{h r}\left(N_{r}\right)$ the fuzzy varying holding cost for the $r^{t h}$ per cycle $=\tilde{C}_{h r} N_{r}-\widetilde{\beta}_{r}$

$\tilde{C}_{\text {or }} \quad$ the fuzzy set up cost per unit item per unit time.

$k_{w r} \quad$ the goal associated to the available warehouse floor space.

$\tau_{r} \quad$ the fraction of the cycle $N_{r}$ in which a stockout does not occur $0<\tau_{r}<N_{r}$.
3. The model analysis:

For periodic review inventory model with zero lead time and instantaneous demand

The following assumptions have been considered:

(i) Replenishment is instantaneous.

(ii) The size of the replenishment order depends upon the number of units demanded from the stock at the review time, then $E\left(x_{r}\right)=\bar{D}_{r} N_{r}(1)$

(iii)No shortages are to be allowed so the buffer stock must be large enough to provide protection against demand fluctuations for a length of time $N+\tau_{r}$, then $x_{u r}=\bar{D}_{r}\left(N_{r}+\tau_{r}\right)(2)$

(iv) Assume that $Q_{m r}=x_{u r}$, thus from Equations (1) and (2):

$Q_{m r}=\bar{D}_{r}\left(N_{r}+\tau_{r}\right)=E\left(x_{r}\right)+s s_{r}$

where $s s_{r}=\bar{D}_{r} \tau_{r}$ is the buffer stock quantity required to absorb demand fluctuations for the $r^{\text {th }}$ item during the cycle $N_{r}$.

The expected total cost for the cycle will be the sum of the expected item cost, expected order cost and the expected varying holding cost, i.e.

$$
E(T C)=\sum_{r=1}^{n}\left[E\left(P C_{r}\right)+E\left(O C_{r}\right)+E\left(H C_{r}\right)\right]
$$


- The expected item cost for the cycle is given by

$$
E\left(P C_{r}\right)=C_{p r} \int_{x=0}^{\infty} x_{r} f\left(x_{r}\right) d x=C_{p r} \bar{x}_{r}
$$

- The expected order cost for the cycle is given by

$$
E\left(O C_{r}\right)=C_{o r}
$$

- The expected varying holding cost for the cycle is given by

$$
E\left(H C_{r}\right)=C_{h r}\left(N_{r}\right) \bar{I}=C_{h r} N_{r}^{-\beta_{r}} \bar{I}
$$

Thus, the expected average amount of inventory is

$$
\begin{gathered}
\bar{I}=Q_{m r}-\frac{E\left(x_{r}\right)}{2}=Q_{m r}-\frac{\bar{D}_{r} N_{r}}{2} \\
\text { Then, } E\left(H C_{r}\right)=\left[C_{h r} N_{r}{ }^{-\beta_{r}}\left(Q_{m r}-\frac{\bar{D}_{r} N_{r}}{2}\right)\right](7)
\end{gathered}
$$

Hence the expected total cost for the cycle is given by,

$E(T C)=\sum_{r=1}^{n}\left[C_{p r} \bar{x}_{r}+C_{o r}+C_{h r} N_{r}{ }^{-\beta_{r}}\left(Q_{m r}-\frac{\bar{D}_{r} N_{r}}{2}\right)\right]$ (8)

The policy variables for this model are $Q_{m r}$ and $N_{r}$. Optimization can be performed if we assume that maximum inventory level during the cycle is related to the average demand during the cycle

$Q_{m r}=\bar{x}_{r} g\left(N_{r}\right)=\bar{D}_{r} N_{r} g\left(N_{r}\right)(9)$

where $g\left(N_{r}\right) \geq 1$ is the relational function just mentioned.

Equation (8) can now be written as

$E\left(T C\left(Q_{m r}, N_{r}\right)\right)$
$=\sum_{r=1}^{n}\left[C_{p r} \bar{x}_{r}+C_{o r}+\frac{C_{h r} \bar{D}_{r} N_{r}{ }^{1-\beta_{r}}}{2}\left[2 g\left(N_{r}\right)-1\right]\right]$ (10)

Consider a limitation on the cost of available warehouse floor space where the items are to be stored, i.e.,

$$
S\left(N_{r}\right)=\sum_{r=1}^{n} S_{r} Q_{m r}=\sum_{r=1}^{n} S_{r} \bar{D}_{r} N_{r} g\left(N_{r}\right) \leq k_{w r}
$$

The problem is to find the optimal maximum inventory level, the optimal time of review (cycle) so as to minimize the expected total average cost function (10) subject to the storage space cost restriction, hence It may written as

Min $E\left(T C\left(Q_{m r}, N_{r}\right)\right)$ for all $r=1,2, \ldots, n$

subject to inequality constraints $S\left(N_{r}\right) \leq k_{w r}$.

Consider the following relationshipg $\left(N_{r}\right)=$ $\frac{N_{r}^{2}+\tau_{r}}{N_{r}{ }^{2}}(12)$

Substituting from Equation (12) into Equation (10) gives

$$
\begin{aligned}
& E(T C)=\sum_{r=1}^{n}\left[C_{p r} \bar{x}+C_{o r}+\frac{C_{h r} \bar{D}_{r} N_{r}{ }^{-\left(\beta_{r}+1\right)}}{2}\left(N_{r}{ }^{2}+2 \tau_{r}\right)\right] \\
& E(T C)=\sum_{r=1}^{n}\left[C_{p r} \bar{x}_{r}+C_{o r}+\frac{C_{h r} \bar{D}_{r} N_{r}{ }^{1-\beta_{r}}}{2}+C_{h r} \bar{D}_{r} N_{r}{ }^{-\left(\beta_{r}+1\right)} \tau_{r}\right](13)
\end{aligned}
$$

The terms $\sum_{r=1}^{n} C_{p r} \bar{x}_{r}, \sum_{r=1}^{n} C_{o r}$ are constants and hence can be postponed without any effect, hence:

$$
\begin{gathered}
\min E(T C)=\sum_{r=1}^{n}\left[\frac{C_{h r} \bar{D}_{r} N_{r}{ }^{1-\beta_{r}}}{2}+C_{h r} \bar{D}_{r} N_{r}{ }^{-\left(\beta_{r}+1\right)} \tau_{r}\right] \\
\text { Subject to: } \sum_{r=1}^{n} \frac{S_{r} \bar{D}_{r} N_{r}}{k_{w r}}+\sum_{r=1}^{n} \frac{S_{r} \bar{D}_{r} \tau_{r}}{N_{r} k_{w r}} \leq 1
\end{gathered}
$$

Applying the geometric programming techniques to Equations (14) and (15), the enlarged pre-dual function could be written in the following form:

$$
\begin{aligned}
& G(\underline{W})=\prod_{r=1}^{n}\left[\begin{array}{c}
\left(\frac{C_{h r} \bar{D}_{r} N_{r}{ }^{1-\beta_{r}}}{2 W_{1 r}}\right)^{W_{1 r}}\left(\frac{C_{h r} \bar{D}_{r} N_{r}{ }^{-\left(\beta_{r}+1\right)} \tau_{r}}{W_{2 r}}\right)^{W_{2 r}} \\
\quad\left(\frac{S_{r} \bar{D}_{r} N_{r}}{k_{w r} W_{3 r}}\right)^{W_{3 r}}\left(\frac{S_{r} \bar{D}_{r} \tau_{r}}{N_{r} k_{w r} W_{4 r}}\right)^{W_{4 r}}
\end{array}\right] \\
& G(\underline{W})=\prod_{r=1}^{n}\left[\begin{array}{l}
\left(\frac{C_{h r} \bar{D}_{r}}{2 W_{1 r}}\right)^{W_{1 r}}\left(\frac{C_{h r} \bar{D}_{r} \tau_{r}}{W_{2 r}}\right)^{W_{2 r}} \\
\times\left(\frac{S_{r} \bar{D}_{r}}{k_{w r} W_{3 r}}\right)^{W_{3 r}}\left(\frac{S_{r} \bar{D}_{r} \tau_{r}}{k_{w r} W_{4 r}}\right)^{W_{4 r}} \\
\times N_{r}\left({ }^{\left.1-\beta_{r}\right) W_{1 r}-\left(\beta_{r}+1\right) W_{2 r}+W_{3 r}-W_{4 r}}\right.
\end{array}\right],
\end{aligned}
$$

Also, the dual function is given by: 


$$
g(\underline{W})=\prod_{r=1}^{n}\left[\begin{array}{l}
\left(\frac{C_{h r} \bar{D}_{r}}{2 W_{1 r}}\right)^{W_{1 r}}\left(\frac{C_{h r} \bar{D}_{r} \tau_{r}}{W_{2 r}}\right)^{W_{2 r}} \\
\times\left(\frac{S_{r} \bar{D}_{r}}{k_{w r} W_{3 r}}\right)^{W_{3 r}}\left(\frac{S_{r} \bar{D}_{r} \tau_{r}}{k_{w r} W_{4 r}}\right)^{W_{4 r}}
\end{array}\right]
$$

where $\underline{W}=W_{j r}, \quad 0<W_{j r}<1, j=1,2,3,4$,

$r=1,2, \ldots, n$ are the weights and can be chosen to yield the following normal and orthogonal conditions:

$$
\begin{aligned}
& W_{1 r}+W_{2 r}=1 \\
& \left(1-\beta_{r}\right) W_{1 r}-\left(\beta_{r}+1\right) W_{2 r}+W_{3 r}-W_{4 r}=0, \\
& \quad r=1,2, \ldots, n .
\end{aligned}
$$

Solving the above Equations, we get:

$$
\left\{\begin{array}{l}
W_{1 r}=\frac{\left(\beta_{r}+1\right)-W_{3 r}+W_{4 r}}{2} \\
W_{2 r}=\frac{\left(1-\beta_{r}\right)+W_{3 r}-W_{4 r}}{2},
\end{array}\right.
$$

Substituting from Equations (17) into Equation (16), we get:

$$
g\left(W_{3 r}, W_{4 r}\right)=\prod_{r=1}^{n}\left[\begin{array}{c}
\left(\frac{C_{h r} \bar{D}_{r}}{\left(\beta_{r}+1\right)-W_{3 r}+W_{4 r}}\right)^{\frac{\left(\beta_{r}+1\right)-W_{3 r}+W_{4 r}}{2}} \times \frac{2 C_{h r} \bar{D}_{r} \tau_{r}}{\times \frac{\left(1-\beta_{r}\right)+W_{3 r}-W_{4 r}}{2}} \\
\left(\frac{S_{r} \bar{D}_{r}}{k_{w r} W_{3 r}}\right)^{W_{3 r}}\left(\frac{S_{r} \bar{D}_{r} \tau_{r}}{k_{w r} W_{4 r}}\right)^{W_{4 r}}
\end{array}\right]
$$

By taking the logarithm of both sides of (18), we have:

$$
\begin{aligned}
& \ln g\left(W_{3 r}, W_{4 r}\right)=\sum_{r=1}^{n}\left[\frac { ( \beta _ { r } + 1 ) - W _ { 3 r } + W _ { 4 r } } { 2 } \left\{\ln \left[C_{h r} \bar{D}_{r}\right]\right.\right. \\
&\left.-\ln \left[\left(\beta_{r}+1\right)-W_{3 r}+W_{4 r}\right]\right\} \\
&+\frac{\left(1-\beta_{r}\right)+W_{3 r}-W_{4 r}}{2}\left\{\ln \left[2 C_{h r} \bar{D}_{r} \tau_{r}\right]\right. \\
&\left.-\ln \left[\left(1-\beta_{r}\right)+W_{3 r}-W_{4 r}\right]\right\} \\
&+W_{3 r}\left\{\ln \left(\frac{S_{r} \bar{D}_{r}}{k_{w r}}\right)-\ln W_{3 r}\right\} \\
&\left.+W_{4 r}\left\{\ln \left(\frac{S_{r} \bar{D}_{r} \tau_{r}}{k_{w r}}\right)-\ln W_{4 r}\right\}\right]
\end{aligned}
$$

Equate the first partial derivatives of $\ln g\left(W_{3 r}, W_{4 r}\right) \quad$ with respect to $W_{3 r}$ and $W_{4 r}$ respectively to zero we can calculate $W_{3 r}{ }^{*}$ and $W_{4 r}{ }^{*}$ which maximize $g\left(W_{3 r}, W_{4 r}\right)$ as follows:

$$
\begin{aligned}
\frac{\partial \ln g\left(W_{3 r}, W_{4 r}\right)}{\partial W_{3 r}}=- & \frac{1}{2}\left\{\ln \left[C_{h r} \bar{D}_{r}\right]-\ln \left[\left(\beta_{r}+1\right)-W_{3 r}+W_{4 r}\right]\right\}+\frac{1}{2} \\
& +\frac{1}{2}\left\{\ln \left[2 C_{h r} \bar{D}_{r} \tau_{r}\right]-\ln \left[\left(1-\beta_{r}\right)+W_{3 r}-W_{4 r}\right]\right\} \\
& -\frac{1}{2}+\left\{\ln \left(\frac{S_{r} \bar{D}_{r}}{k_{w r}}\right)-\ln W_{3 r}\right\}-1=0
\end{aligned}
$$

Then we get,

$$
\begin{array}{r}
W_{3 r}{ }^{2}=\left(\frac{2 \tau\left[\left(\beta_{r}+1\right)-W_{3 r}+W_{4 r}\right]}{\left[\left(1-\beta_{r}\right)+W_{3 r}-W_{4 r}\right]}\right)\left(\frac{S_{r} \bar{D}_{r}}{k_{w r} e}\right)^{2} \\
\frac{\partial \ln g\left(W_{3 r}, W_{4 r}\right)}{\partial W_{4 r}}=\frac{1}{2}\left\{\ln \left[C_{h r} \bar{D}_{r}\right]-\ln \left[\left(\beta_{r}+1\right)-W_{3 r}+W_{4 r}\right]\right\} \\
-\frac{1}{2}-\frac{1}{2}\left\{\ln \left[2 C_{h r} \bar{D}_{r} \tau_{r}\right]-\ln \left[\left(1-\beta_{r}\right)+W_{3 r}-W_{4 r}\right]\right\}+\frac{1}{2} \\
+\left\{\ln \left(\frac{S_{r} \bar{D}_{r} \tau_{r}}{k_{w r}}\right)-\ln W_{4 r}\right\}-1=0
\end{array}
$$

Then we get,

$$
W_{4 r}^{2}=\left(\frac{\left[\left(1-\beta_{r}\right)+W_{3 r}-W_{4 r}\right]}{2 \tau\left[\left(\beta_{r}+1\right)-W_{3 r}+W_{4 r}\right]}\right)\left(\frac{S_{r} \bar{D}_{r} \tau_{r}}{k_{w r} e}\right)^{2}
$$

Multiplying Equations (19) and (20) and simplifying them, we get:

$$
W_{3 r} W_{4 r}=\left(\frac{S_{r} \bar{D}_{r}}{k_{w r} e}\right)\left(\frac{S_{r} \bar{D}_{r} \tau_{r}}{k_{w r} e}\right)
$$

$$
\begin{gathered}
W_{3 r} W_{4 r}=B_{1}{ }^{2} \tau_{r}, \quad \text { where } B_{1}=\left(\frac{S_{r} \bar{D}_{r}}{k_{w r} e}\right) \\
\begin{aligned}
& f\left(W_{3 r}\right)=\left[\left(1-\beta_{r}\right)+W_{3 r}-B_{1}{ }^{2} \tau_{r} W_{3 r}{ }^{-1}\right] W_{3 r}{ }^{2} \\
&- \\
&+2 \tau_{r}\left[\left(\beta_{r}+1\right)-W_{3 r}\right. \\
&\left.+B_{1}{ }^{2} \tau_{r} W_{3 r}{ }^{-1}\right] B_{1}{ }^{2}=0 \\
& f\left(W_{4 r}\right)= 2 \tau_{r}\left[\left(\beta_{r}+1\right)-B_{1}{ }^{2} \tau_{r} W_{4 r}{ }^{-1}+W_{4 r}\right] W_{4 r}{ }^{2} \\
&-\left[\left(1-\beta_{r}\right)+B_{1}{ }^{2} \tau_{r} W_{4 r}{ }^{-1}\right. \\
&\left.-W_{4 r}\right] B_{1} \tau_{r}{ }^{2}=0
\end{aligned}
\end{gathered}
$$

It could be easily proved that $\mathrm{f}_{j}(0)<1$ and $\mathrm{f}_{j}(1)>0, \forall \mathrm{j}=3,4$, and this means that there exists a root $w_{\mathrm{jr}} \in(0,1), \mathrm{j}=3,4$. Numerical 
methods (such as dichotomous search) could be used to calculate these roots for eachitem.

Now it is to be verified that any roots $\mathrm{W}_{3 \mathrm{r}}^{*}, \mathrm{~W}_{3 \mathrm{r}}^{*}$ calculated from Equation $(22)$ and (23) maximizeg $\left(\mathrm{W}_{3 \mathrm{r}}^{*}, \mathrm{~W}_{3 \mathrm{r}}^{*}\right)$ respectively. Apply the following conditions:

$\frac{\partial^{2} \ln g\left(W_{3 r}, W_{4 r}\right)}{\partial W_{3 r}{ }^{2}}=-\frac{1}{4}\left[\frac{1}{W_{1 r}}+\frac{1}{W_{2 r}}\right]-\frac{1}{W_{3 r}}<0$

$\frac{\partial^{2} \ln g\left(W_{3 r}, W_{4 r}\right)}{\partial W_{4 r}^{2}}=-\frac{1}{4}\left[\frac{1}{W_{1 r}}+\frac{1}{W_{2 r}}\right]-\frac{1}{W_{4 r}}<0$

$\frac{\partial^{2} \ln g\left(W_{3 r}, W_{4 r}\right)}{\partial W_{3 r} \partial W_{4 r}}=\frac{\partial^{2} \ln g\left(W_{3 r}, W_{4 r}\right)}{\partial W_{4 r} \partial W_{3 r}}=\frac{1}{4}\left[\frac{1}{W_{1 r}}+\frac{1}{W_{2 r}}\right]>0$

It is clear that: $\left[\frac{\partial^{2} \ln g\left(W_{3 r}, W_{4 r}\right)}{\partial W_{j r}{ }^{2}}\right]<0$

Therefore from the Hesian matrix we have:

$$
\begin{aligned}
\Delta=\left(\frac{\partial^{2} \ln g\left(W_{3 r}, W_{4 r}\right)}{\partial W_{3 r} \partial W_{4 r}}\right)^{2} & -\left(\frac{\partial^{2} \ln g\left(W_{3 r}, W_{4 r}\right)}{\partial W_{3 r}{ }^{2}}\right)\left(\frac{\partial^{2} \ln g\left(W_{3 r}, W_{4 r}\right)}{\partial W_{4 r}{ }^{2}}\right) \\
& =-\frac{1}{4}\left[\frac{1}{W_{1 r}}+\frac{1}{W_{2 r}}\right]\left[\frac{1}{W_{3 r}}+\frac{1}{W_{4 r}}\right]-\frac{1}{W_{3 r} W_{4 r}}<0
\end{aligned}
$$

Therefore the roots $W_{3 r}^{*}, W_{4 r}^{*}$ calculated from Equations (22), (23) maximize the dual function $g\left(W_{3 r}, W_{4 r}\right)$. Hence the optimal solution is $W_{j r}^{*}, j=1,2,3,4$ where $W_{1 r}^{*}, W_{2 r}^{*}$ are calculated by substituting the values of $W_{3 r}^{*}$ and $W_{4 r}^{*}$ in Equation (17).

To find the optimal review time (cycle) $N_{r}{ }^{*}$, the optimal maximum inventory level $Q_{m r}^{*}$ and the minimum expected total cost $\min E(T C)$ use the following relations due to Duffin and Peterson's theorem (1966) of geometric programming as follows:

$\frac{C_{h r} \bar{D}_{r} N_{r}{ }^{1-\beta_{r}}}{2}=W_{1 r}^{*} g\left(W_{3 r}^{*}, W_{4 r}^{*}\right)$ and

$C_{h r} \bar{D}_{r} N_{r}{ }^{-\left(\beta_{r}+1\right)} \tau_{r}=W_{2 r}^{*} g\left(W_{3 r}^{*}, W_{4 r}^{*}\right)$

hence,

$N_{r}^{*}=\left(\frac{2 \tau_{r} W_{1 r}^{*}}{W_{2 r}^{*}}\right)^{\frac{1}{2}}$

$Q_{m r}^{*}=\bar{D}_{r} N^{*}{ }_{r} g\left(N_{r}\right)=\bar{D}_{r} N^{*}{ }_{r}\left(\frac{N^{*}{ }_{r}{ }^{2}+\tau_{r}}{N^{*}{ }_{r}{ }^{2}}\right)$

$Q_{m r}^{*}=\bar{D}_{r} N^{*}{ }_{r}\left[1+\frac{\tau_{r}}{N^{*}{ }^{2}}\right]$

Substituting the value of $N_{r}^{*}$ in Equation (13), after restore the constant terms, we get:

$\min E(T C)=\sum_{r=1}^{n}\left[\begin{array}{c}C_{p r} \bar{x}_{r}+C_{o r}+\frac{C_{h r} \bar{D}_{r} N^{*}{ }_{r}{ }^{1-\beta_{r}}}{2} \\ +C_{h r} \bar{D}_{r} N^{*}{ }_{r}{ }^{-\left(\beta_{r}+1\right)} \tau_{r}\end{array}\right]$

$\min E(T C)=\sum_{r=1}^{n}\left[\begin{array}{c}C_{p r} \bar{x}_{r}+C_{o r}+\frac{C_{h r} \bar{D}_{r} N^{*}{ }_{r}{ }^{-\left(\beta_{r}+1\right)}}{2} \\ \left(N^{*}{ }^{2}+2 \tau_{r}\right)\end{array}\right]$

which is the optimal minimum expected total cost.

\section{The model when All parameters are Fuzzy Numbers}

The inventory cost coefficients, elasticity parameters and other coefficients in the model are fuzzy in nature. Therefore, the decision variables and the objective function should be fuzzy as well, and we are interested in deriving the membership functions of $E(T C)$ by solving the model via geometric programming According to the assumptions, we have the following formulation for the fuzzy inventory model:

$$
E(\widetilde{T C})=\sum_{r=1}^{n}\left[\begin{array}{c}
\tilde{C}_{p r} \bar{x}_{r}+\tilde{C}_{o r}+\frac{\tilde{C}_{h r} \bar{D}_{r} N_{r}{ }^{1-\widetilde{\beta}_{r}}}{2} \\
+\tilde{C}_{h r} \bar{D}_{r} N_{r}{ }^{\left.-\widetilde{\beta}_{r}+1\right)} \tilde{\tau}_{r}
\end{array}\right]
$$

The terms $\sum_{r=1}^{n} \tilde{C}_{p r} \bar{x}_{r}, \quad \sum_{r=1}^{n} \tilde{C}_{o r}$ are constants and hence can be postponed Restores without any effect, hence Equation (27) tends to 


$$
\begin{gathered}
E(\widetilde{T C})=\sum_{r=1}^{n}\left[\frac{\tilde{C}_{h r} \bar{D}_{r} N_{r}{ }^{1-\tilde{\beta}_{r}}}{2}+\tilde{C}_{h r} \bar{D}_{r} N_{r}{ }^{-\left(\widetilde{\beta}_{r}+1\right)} \tilde{\tau}_{r}\right] \\
\text { Subject to: } \sum_{r=1}^{n} \frac{\tilde{S}_{r} \bar{D}_{r} N_{r}}{\tilde{k}_{w r}}+\sum_{r=1}^{n} \frac{\tilde{S}_{r} \bar{D}_{r} \tilde{\tau}_{r}}{N_{r} \tilde{k}_{w r}} \leq 1
\end{gathered}
$$

To solve this inventory model using geometric programming technique, we should find the right and the left shape functions of the objective function and decision variables, by find the upper bound and the lower bound of the objective function, i.e. $\widetilde{T C}^{L}(\propto)$ and $\widetilde{T C}^{R}(\propto)$. Recall that $\widetilde{T C}^{L}(\propto)$ and $\widetilde{T C}^{R}(\propto)$ represent the largest and the smallest values (The left and right $\propto$ cuts)of the optimal objective function $\widetilde{T C}(\propto)$.

Consider the model when all parameters are triangular fuzzy numbers (TFN) as given below

$C_{p r}=\left(C_{p r}-a_{1 r}, C_{p r}, C_{p r}+a_{2 r}\right)$,

$C_{o r}=\left(C_{o r}-a_{3 r}, C_{o r}, C_{o r}+a_{4 r}\right)$,

$C_{h r}=\left(C_{h r}-a_{5 r}, C_{h r}, C_{h r}+a_{6 r}\right)$,

$S_{r}=\left(S_{r}-a_{7 r}, S_{r}, S_{r}+a_{8 r}\right)$,

$\beta_{r}=\left(\beta_{r}-a_{9 r}, \beta_{r}, \beta_{r}+a_{10 r}\right)$,

$K_{w r}=\left(K_{w r}-a_{11 r}, K_{w r}, K_{w r}+a_{12 r}\right)$

and $\tau_{r}=\left(\tau_{r}-a_{13 r}, \tau_{r}, \tau_{r}+a_{14 r}\right)$.

where $a_{i r}, i=1,2, \ldots \ldots, 14$ are arbitrary positive numbers under the following restrictions:

$0 \leq a_{1 r} \leq C_{p r}, a_{2 r} \geq 0,0 \leq a_{3 r} \leq C_{o r}, a_{4 r} \geq 0$,

$0 \leq a_{5 r} \leq C_{h r}, a_{6 r} \geq 0,0 \leq a_{7 r} \leq S_{r}, a_{8 r} \geq 0$,

$0 \leq a_{9 r} \leq \beta_{r}, a_{10 r} \geq 0,0 \leq a_{11 r} \leq K_{w r}, a_{12 r} \geq 0$

and $\quad 0 \leq a_{13 r} \leq \tau_{r}, a_{14 r} \geq 0$

The left and right limits of $\propto$ cuts of $C_{p r}, C_{o r}, C_{h r}, S_{r}, \beta_{r}, K_{w r}$ and $\tau_{r}$ are given by

$\tilde{C}_{p r_{L}}(\propto)=C_{p r}-(1-\propto) a_{1 r} \tilde{C}_{p r_{R}}(\propto)=C_{p r}+(1-\propto) a_{2 r}$

$\tilde{C}_{o r_{L}}(\propto)=C_{o r}-(1-\propto) a_{3 r}, \tilde{C}_{o r_{R}}(\propto)=C_{o r}+(1-\propto) a_{4 r}$,

$$
\begin{aligned}
& \tilde{C}_{h r_{L}}(\propto)=C_{h r}-(1-\propto) a_{5 r}, \tilde{C}_{h r_{R}}(\propto)=C_{h r}+(1-\propto) a_{6 r} \\
& \tilde{S}_{r_{L}}(\propto)=S_{r}-(1-\propto) a_{7 r}, \tilde{S}_{r_{R}}(\propto)=S_{r}+(1-\propto) a_{8 r} \\
& \tilde{\beta}_{r_{L}}(\propto)=\beta_{r}-(1-\propto) a_{9 r}, \tilde{\beta}_{r_{R}}(\propto)=\beta_{r}+(1-\propto) a_{10 r} \\
& \widetilde{K}_{w r_{L}}(\propto)=K_{w r}-(1-\propto) a_{11 r}, \widetilde{K}_{w r_{R}}(\propto)=K_{w r}+(1-\propto) a_{12 r} \\
& \text { and } \tilde{\tau}_{r_{L}}(\propto)=\tau_{r}-(1-\propto) a_{13 r}, \tilde{\tau}_{r_{R}}(\propto)=\tau_{r}(1-\propto) a_{14 r} .
\end{aligned}
$$

\section{where}

$$
\begin{aligned}
& \tilde{C}_{p r}=C_{p r}+\frac{1}{4}\left(a_{2 r}-a_{1 r}\right), \tilde{C}_{o r}=C_{o r}+\frac{1}{4}\left(a_{4 r}-a_{3 r}\right), \\
& \tilde{C}_{h r}=C_{h r}+\frac{1}{4}\left(a_{6 r}-a_{5 r}\right), \tilde{S}_{r}=S_{r}+\frac{1}{4}\left(a_{8 r}-a_{7 r}\right) \\
& \tilde{\beta}_{r}=\beta_{r}+\frac{1}{4}\left(a_{10 r}-a_{9 r}\right), \widetilde{K}_{w r}=K_{w r}+\frac{1}{4}\left(a_{12 r}-a_{11 r}\right) \\
& \text { and } \tilde{\tau}_{r}=\tau_{r}+\frac{1}{4}\left(a_{14}-a_{13}\right) .
\end{aligned}
$$

Using approximated value of TFN which observe in Figure 2.

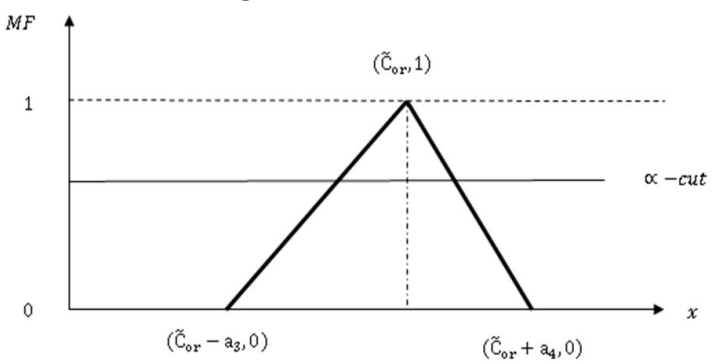

Figure 2: Order cost as triangular fuzzy number By using the Geometric Programming approach, the above fuzzy system of Equations (28), (29) reduces to

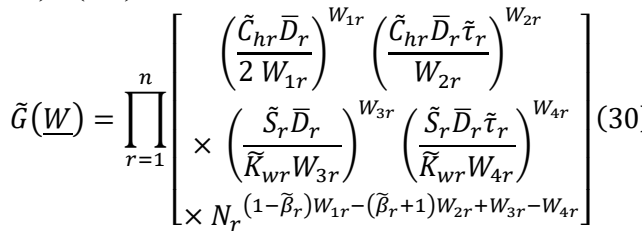

hence,

$$
=\prod_{r=1}^{n}\left[\begin{array}{c}
\left(\frac{\tilde{C}_{h r} \bar{D}_{r}}{\left(\tilde{\beta}_{r}+1\right)-W_{3 r}+W_{4 r}}\right)^{\frac{\left(\tilde{\beta}_{r}+1\right)-W_{3 r}+W_{4 r}}{2}} \\
\times\left(\frac{2 \tilde{C}_{h r} \bar{D}_{r} \tilde{\tau}_{r}}{\left(1-\tilde{\beta}_{r}\right)+W_{3 r}-W_{4 r}}\right)^{\frac{\left(1-\tilde{\beta}_{r}\right)+W_{3 r}-W_{4 r}}{2}} \\
\times\left(\frac{\tilde{S}_{r} \bar{D}_{r}}{\widetilde{K}_{w r} W_{3 r}}\right)^{W_{3 r}}\left(\frac{\tilde{S}_{r} \bar{D}_{r} \tilde{\tau}_{r}}{\widetilde{K}_{w r} W_{4 r}}\right)^{W_{4 r}}
\end{array}\right]
$$

Due to Duffin and Peterson's theorem (1966), the optimal values are given by:

$N_{r}^{*}=\left(\frac{2 \tilde{\tau}_{r} W_{1 r}^{*}}{W_{2 r}^{*}}\right)^{\frac{1}{2}}$ 


$$
Q_{m r}^{*}=\bar{D}_{r} N_{r}^{*}\left[1+\frac{\tilde{\tau}_{r}}{N^{*}{ }_{r}^{2}}\right]
$$

Then we can determine the optimal minimum expected total cost as follows:

$\min E(\widetilde{T C})=\sum_{r=1}^{n}\left[\begin{array}{c}\widetilde{C}_{p r} \bar{x}_{r}+\widetilde{C}_{o r}+\frac{\widetilde{C}_{h r} \bar{D}_{r} N_{r}{ }_{r}{ }^{1-\widetilde{\beta}_{r}}}{2} \\ +\widetilde{C}_{h r} \bar{D}_{r} N^{*}{ }_{r}{ }^{-\left(\widetilde{\beta}_{r}+1\right)} \tilde{\tau}_{r}\end{array}\right]$

5. The Model with Continuous Distributions Suppose that the demand for a particular item follows some Continuous distribution such as:

The demand follows uniform distribution over range from zero to $b$ then, $\min E(T C)$

$=\sum_{r=1}^{n}\left[C_{p r} \frac{Q_{m}{ }^{2}}{2 b}+C_{o r}+\frac{C_{h r} \bar{D}_{r} N^{*}{ }_{r}{ }^{-\left(\beta_{r}+1\right)}}{2}\left(N^{*}{ }_{r}{ }^{2}+2 \tau_{r}\right)\right]$

The demand follows negative exponential distribution when $0<x<Q_{m r}, \theta>0$ then, $\min E(T C)=\sum_{r=1}^{n}\left[\begin{array}{l}\frac{C_{p r}}{\theta}\left(1-e^{-\theta Q_{m r}}\left(1+\theta Q_{m r}\right)\right)+C_{o r} \\ +\frac{C_{h r} \bar{D}_{r} N^{*}{ }_{r}{ }^{-\left(\beta_{r}+1\right)}}{2}\left(N^{*}{ }_{r}{ }^{2}+2 \tau_{r}\right)\end{array}\right]$

The demand follows exponential distribution when $x \geq 0$ then,

$\min E(T C)=\sum_{r=1}^{n}\left[\begin{array}{l}\frac{C_{p r}}{\theta}\left(\theta^{2}-\theta e^{-\frac{Q_{m r}}{\theta}}\left(\theta+Q_{m r}\right)\right)+C_{o r} \\ +\frac{C_{h r} \bar{D}_{r} N^{*}{ }_{r}-\left(\beta_{r}+1\right)}{2}\left(N^{*}{ }_{r}{ }^{2}+2 \tau_{r}\right)\end{array}\right]$

The demand follows Cauchy distribution when $-\infty<x<\infty$ then, $\min E(T C)=\sum_{r=1}^{n}\left[\begin{array}{c}\frac{C_{p r}}{2 \pi} \ln \left(1+Q_{m}{ }^{2}\right)+C_{o r} \\ +\frac{C_{h r} \bar{D}_{r} N^{*}{ }_{r}-\left(\beta_{r}+1\right)}{2}\left(N^{*}{ }^{2}+2 \tau_{r}\right)\end{array}\right]$

\section{The demand follows Weibull distribution} when the variable $\mathrm{x}$ and the parameters $\eta$ and $\sigma$ all are positive real numbers then,

$\min E(T C)=\sum_{r=1}^{n}\left[\begin{array}{c}C_{p r}\left(1-e^{-\left(\frac{x}{\sigma}\right)^{\eta}}\right)+C_{o r} \\ +\frac{C_{h r} \bar{D}_{r} N^{*}{ }_{r}-\left(\beta_{r}+1\right)}{2}\left(N^{*}{ }^{2}+2 \tau_{r}\right)\end{array}\right]$

The demand follows Rayleigh distribution when the variablexis real positive valuesand $\alpha$ a real positive parameter then, $\min E(T C)=\sum_{r=1}^{n}\left[\begin{array}{c}C_{p r}\left(Q_{m} e^{-\frac{x^{2}}{2 \alpha^{2}}}-\alpha \sqrt{\frac{\pi}{2}} e^{-\frac{x}{\sqrt{2} \alpha}}\right)+C_{o r} \\ +\frac{C_{h r} \bar{D}_{r} N^{*}{ }_{r}-\left(\beta_{r}+1\right)}{2}\left(N^{*}{ }_{r}{ }^{2}+2 \tau_{r}\right)\end{array}\right]$

The demand follows triangular distribution when the variable $\mathrm{x}$ is bounded to the interval $x-\Gamma \leq \mathrm{x} \leq x+\Gamma$ and the location and scale parameters $\gamma$ and $\gamma=\Gamma$ $(\Gamma>0)$ all are real numbers. $\min E(T C)$

$=\sum_{r=1}^{n}\left[\begin{array}{c}\frac{C_{p r}}{2 \Gamma^{2}}\left(\Gamma Q_{m}{ }^{2}-\frac{1}{3}\left(\gamma^{2}|\gamma| \frac{\left(Q_{m}-\gamma\right)^{2}\left(2 Q_{m}+\gamma\right)}{\operatorname{sign}\left(Q_{m}-\gamma\right)}\right)\right) \\ +C_{o r}+\frac{C_{h r} \bar{D}_{r} N^{*}{ }_{r}{ }^{-\left(\beta_{r}+1\right)}}{2}\left(N^{*}{ }_{r}{ }^{2}+2 \tau_{r}\right)\end{array}\right]$

\section{Numerical example}

A businessman decides to open a Boutique, say XYZ, in Egypt, say, where he wishes to exclusively showcase indigenously made fabrics of three items (e.g. ties, shirts and trousers) of India. As the businessman has to travel to the places where these bolts of fabrics are made to collect his orders, he wishes to follow a periodic review "order up to $Q_{m}$ ". Sufficient information is not available for this kind of a business. But from what is available, he estimates that the parameters are given in Table 1, Table2.The demand is occurs at the begging of the cycle. Also the optimal solutions of the crisp environment and triangular fuzzy number TFN are given in Tables 3, 4, 5, 6, 7, 8 and 9.

It is desired to determine to optimal values $Q_{m}{ }^{*}, N^{*}$ and the minimum total cost in the following cases:

When demand follows

1- Uniform distribution for $0 \leq x \leq b$,

2- negative exponential distribution for $x \geq 0$.

3- Exponential distribution for $x \geq 0$.

4- Cauchy Distribution for $-\infty<x<\infty$

5- Weibull Distribution for $x \geq 0$.

6- Rayleigh Distribution for $x \geq 0$.

7- Triangular Distribution for $\mathrm{x}-\Gamma \leq \mathrm{x} \leq$ $\mathrm{x}+\Gamma$. 


\begin{tabular}{|c|c|c|c|}
\hline Parameters & Item 1 & Item 2 & Item 3 \\
\hline $\boldsymbol{\beta}_{r}$ & $(0,1)$ & $(0,1)$ & $(0,1)$ \\
\hline$C_{p r}$ & 11 & 9 & 14 \\
\hline$C_{o r}$ & 15 & 13 & 17 \\
\hline$\overline{\bar{D}}$ & 7 & 5 & 9 \\
\hline$S_{r}$ & 5 & 4 & 6 \\
\hline$C_{h r}$ & 4 & 3 & 5 \\
\hline$K_{w r}$ & 102 & 51.9 & 36.2 \\
\hline$\tau_{r}$ & 1.9 & 1.5 & 0.9 \\
\hline$b_{r}$ & 45 & 50 & 55 \\
\hline$\theta_{r}$ & 6 & 5 & 7 \\
\hline$\eta_{r}$ & 7 & 4.5 & 8.5 \\
\hline$\sigma_{r}$ & 6 & 5 & 7 \\
\hline$\alpha_{r}$ & 4 & 3 & 6.5 \\
\hline$\gamma_{r}=\Gamma_{r}$ & 13 & 11 & 14 \\
\hline
\end{tabular}

Table 1: Crisp values of the parameters

\begin{tabular}{|c|c|c|c|}
\hline Parameters & Item 1 & Item 2 & Item 3 \\
\hline$\tilde{C}_{p r}$ & $(8,11,12)$ & $(7,9,10)$ & $(12,14,15)$ \\
\hline$\tilde{C}_{o r}$ & $(12,15,16)$ & $(10,13,15)$ & $(14,17,19)$ \\
\hline$\tilde{S}_{r}$ & $(3,5,5.5)$ & $(3,4,4.5)$ & $(4,6,7)$ \\
\hline$\tilde{C}_{h r}$ & $(2,4,4.5)$ & $(2,3,3.3)$ & $(3,5,6)$ \\
\hline$\widetilde{K}_{w r}$ & $(94,102,104)$ & $(42.8,51.9,53)$ & $(32,36.2,40)$ \\
\hline$\tilde{\tau}_{r}$ & $(1.7,1.9,1.95)$ & $(1.2,1.5,1.7)$ & $(0.8,0.9,0.95)$ \\
\hline
\end{tabular}

Table 2:Input imprecise data for shape parameters

\begin{tabular}{|c|c|c|c|c|c|c|c|c|c|c|c|c|c|}
\hline \multirow{2}{*}{$\boldsymbol{\beta}_{r}$} & \multicolumn{4}{|c|}{ Item 1} & \multicolumn{4}{|c|}{ Item 2} & \multicolumn{4}{|c|}{ Item 3} & \multirow{2}{*}{$\operatorname{Min} E(T C)$} \\
\hline & $N_{1}$ & $Q_{m 1}$ & $E(T C)_{1}$ & $\begin{array}{l}E\left(T C_{1}\right)= \\
E(T C) / Q_{m 1}\end{array}$ & $N_{2}$ & $Q_{m 2}$ & $E(T C)_{2}$ & $\begin{array}{l}E\left(T C_{2}\right)= \\
E(T C) / Q_{m 2}\end{array}$ & $N_{3}$ & $Q_{m 3}$ & $E(T C)_{3}$ & $\begin{array}{l}E\left(T C_{3}\right)= \\
E(T C) / Q_{m 3}\end{array}$ & \\
\hline 0.1 & 2.20956 & 21.4862 & 145.177 & 6.75673 & 1.94171 & 13.5711 & 69.517 & 5.12242 & S1.25852 & 17.7628 & 143.952 & 8.10412 & 19.98327 \\
\hline 0.2 & 2.35372 & 22.1267 & 143.435 & 6.48246 & 2.0699 & 13.9729 & 68.7665 & 4.92143 & 1.31598 & 17.9989 & 143.417 & 7.96809 & 19.37198 \\
\hline 0.3 & 2.50934 & 22.8656 & 141.914 & 6.20646 & 2.20829 & 14.4377 & 67.9958 & 4.70959 & 1.37575 & 18.2694 & 142.491 & 7.7994 & 18.71545 \\
\hline 0.4 & 2.67792 & 23.712 & 140.808 & 5.93828 & 2.35823 & 14.9715 & 67.2824 & 4.49403 & 1.43795 & 18.5746 & 141.225 & 7.60313 & 18.03544 \\
\hline 0.5 & 2.86112 & 24.6763 & 140.336 & 5.68706 & 2.52129 & 15.5811 & 66.7151 & 4.28179 & 1.50271 & 18.9147 & 139.683 & 7.38489 & 17.35374 \\
\hline 0.6 & 3.06076 & 25.7707 & 140.739 & 5.46121 & 2.69915 & 16.2744 & 66.3941 & 4.07967 & 1.57017 & 19.2902 & 137.936 & 7.15058 & 16.69146 \\
\hline 0.7 & 3.27882 & 27.0081 & 142.287 & 5.26832 & 2.89366 & 17.0602 & 66.4322 & 3.89399 & 1.64046 & 19.7018 & 136.065 & 6.90622 & 16.06853 \\
\hline 0.8 & 3.51739 & 28.4029 & 145.278 & 5.1149 & 3.10684 & 17.9482 & 66.9553 & 3.73047 & 1.71372 & 20.15 & 134.154 & 6.65773 & 15.5031 \\
\hline 0.9 & 3.77876 & 29.971 & 150.045 & 5.00635 & 3.34082 & 18.949 & 68.104 & 3.59406 & 1.79009 & 20.6357 & 132.291 & 6.4108 & 15.01121 \\
\hline$\widetilde{\boldsymbol{\beta}}_{r}$ & $\widetilde{N}_{1}$ & $\widetilde{Q}_{m 1}$ & $E(\widetilde{T C})_{1}$ & $\begin{array}{l}E\left(\widetilde{T C}_{1}\right)= \\
E(\widetilde{T C})_{1} / Q_{m 1}\end{array}$ & $\widetilde{N}_{2}$ & $\widetilde{Q}_{m 2}$ & $E(\widetilde{T C})_{2}$ & $\begin{array}{l}E\left(\widetilde{T C} \widetilde{C}_{2}\right) \\
E(\widetilde{T C})_{2} / Q_{m 2}\end{array}$ & $\widetilde{N}_{3}$ & $\widetilde{Q}_{m 3}$ & $E(\widetilde{T C})_{3}$ & $\begin{array}{l}E\left(\widetilde{T C}_{3}\right)= \\
E(\widetilde{T C})_{3} / Q_{m 3}\end{array}$ & $\operatorname{Min} E(\widetilde{T C})$ \\
\hline 0.1 & 2.1807 & 21.2434 & 133.814 & 6.29908 & 1.9058 & 13.3988 & 58.8633 & 4.39317 & 1.2585 & 17.6732 & 134.88 & 7.63188 & 18.32413 \\
\hline 0.2 & 2.3262 & 21.8879 & 132.531 & 6.055 & 2.0316 & 13.7881 & 58.0367 & 4.2092 & 1.3171 & 17.9183 & 134.489 & 7.50568 & 17.76988 \\
\hline 0.3 & 2.4836 & 22.6347 & 131.462 & 5.80801 & 2.16725 & 14.2392 & 57.146 & 4.01329 & 1.3782 & 18.1992 & 133.73 & 7.34813 & 17.16943 \\
\hline 0.4 & 2.65465 & 23.4938 & 130.794 & 5.56719 & 2.31419 & 14.7578 & 56.2563 & 3.81197 & 1.44179 & 18.5161 & 132.652 & 7.16416 & 16.54332 \\
\hline 0.5 & 2.84117 & 24.477 & 130.739 & 5.34133 & 2.4739 & 15.3506 & 55.4424 & 3.61174 & 1.50813 & 18.8694 & 131.317 & 6.95924 & 15.91231 \\
\hline 0.6 & 3.04522 & 25.5979 & 131.539 & 5.13867 & 2.64805 & 16.0253 & 54.7884 & 3.41886 & 1.57733 & 19.2599 & 129.795 & 6.73913 & 15.29666 \\
\hline 0.7 & 3.2691 & 26.8716 & 133.464 & 4.96674 & 2.8385 & 16.7906 & 54.3883 & 3.23921 & 1.6495 & 19.6881 & 128.164 & 6.5097 & 14.71565 \\
\hline 0.8 & 3.5151 & 28.3145 & 136.821 & 4.83218 & 3.047 & 17.656 & 54.3466 & 3.07809 & 1.7249 & 20.155 & 126.508 & 6.27675 & 14.18702 \\
\hline 0.9 & 3.7859 & 29.9447 & 141.957 & 4.74063 & 3.2762 & 18.6319 & 54.7792 & 2.94008 & 1.8036 & 20.6613 & 124.915 & 6.04584 & 13.72655 \\
\hline
\end{tabular}


Table 3: The results of crisp and fuzzy values for uniform distribution

\begin{tabular}{|c|c|c|c|c|c|c|c|c|c|c|c|c|c|}
\hline \multirow[b]{2}{*}{$\boldsymbol{\beta}_{r}$} & \multicolumn{4}{|c|}{ Item 1} & \multicolumn{4}{|c|}{ Item 2} & \multicolumn{4}{|c|}{ Item 3} & \multirow{2}{*}{$\operatorname{Min} E(T C)$} \\
\hline & $N_{1}$ & $Q_{m 1}$ & $E(T C)_{1}$ & $\begin{array}{l}E\left(T C_{1}\right)= \\
E(T C) / Q_{m}\end{array}$ & $N_{2}$ & $Q_{m 2}$ & $E(T C)_{2}$ & $\begin{array}{l}E\left(T C_{2}\right)= \\
E(T C) / Q_{m}\end{array}$ & $N_{3}$ & $Q_{m 3}$ & $E(T C)_{3}$ & $\begin{array}{l}E\left(T C_{3}\right)= \\
E(T C) / Q_{m}\end{array}$ & \\
\hline 0.1 & 2.20956 & 21.4862 & 96.2276 & 4.47858 & 1.94171 & 13.5711 & 52.8995 & 3.89794 & 1.25852 & 17.7628 & 105.795 & 5.95599 & 14.33251 \\
\hline 0.2 & 2.35372 & 22.1267 & 91.4138 & 4.13139 & 2.0699 & 13.9729 & 51.0424 & 3.65297 & 1.31598 & 17.9989 & 104.186 & 5.78844 & 13.5728 \\
\hline 0.3 & 2.50934 & 22.8656 & 86.2357 & 3.77142 & 2.20829 & $\begin{array}{l}14.4377 \\
\end{array}$ & 48.951 & 3.39049 & 1.37575 & 18.2694 & 102.011 & 5.58367 & 12.74558 \\
\hline 0.4 & 2.67792 & 23.712 & 80.7934 & 3.40728 & 2.35823 & 14.9715 & 46.6678 & 3.11711 & 1.43795 & 18.5746 & 99.314 & 5.34677 & 11.87116 \\
\hline 0.5 & 2.86112 & 24.6763 & 75.1877 & 3.04695 & 2.52129 & 15.5811 & \begin{tabular}{|l|l|}
44.238 \\
\end{tabular} & 2.83921 & 1.50271 & 18.9147 & 96.1491 & 5.08331 & 10.96947 \\
\hline $\begin{array}{ll}0.6 \\
\end{array}$ & 3.06076 & 25.7707 & 69.5184 & 2.69758 & 2.69915 & $\begin{array}{l}16.2744 \\
\end{array}$ & 41.7085 & 2.56283 & 1.57017 & 19.2902 & 92.5765 & 4.79914 & 10.05955 \\
\hline 0.7 & 3.27882 & 27.0081 & 63.8824 & 2.36531 & 2.89366 & 17.0602 & 39.1272 & 2.29348 & 1.64046 & 19.7018 & 88.6626 & 4.50023 & 9.15902 \\
\hline 0.8 & 3.51739 & 28.4029 & 58.3715 & 2.05512 & 3.10684 & 17.9482 & 36.5414 & 2.03594 & 1.71372 & 20.15 & 84.4778 & 4.19244 & 8.06171 \\
\hline 0.9 & 3.77876 & 29.971 & 53.0699 & 1.77071 & 3.34082 & 18.949 & 33.9973 & 1.79415 & 1.79009 & 20.6357 & 80.0945 & 3.88135 & 7.44621 \\
\hline$\widetilde{\boldsymbol{\beta}}_{r}$ & $\widetilde{N}_{1}$ & $\widetilde{Q}_{m 1}$ & $E(\widetilde{T C})_{1}$ & $\begin{array}{l}E\left(\widetilde{T C}_{1}\right)= \\
E(\widetilde{T C})_{1} / Q_{n}\end{array}$ & $\tilde{N}_{2}$ & $\tilde{Q}_{m 2}$ & $E(\widetilde{T C})_{2}$ & $\begin{array}{l}E(\widetilde{T \widetilde{T}})_{2}= \\
E(\widetilde{T C})_{2} / Q_{1}\end{array}$ & $\widetilde{N}_{3}$ & $\widetilde{Q}_{m 3}$ & $E(\widetilde{T C})_{3}$ & $\begin{array}{l}\left.E(\widetilde{T \widetilde{T}})_{3}\right)= \\
E(\widetilde{T C})_{3} / Q_{n}\end{array}$ & $\operatorname{Min} E(\widetilde{T C})$ \\
\hline 0.1 & 2.1807 & 21.2434 & 88.5292 & 4.16737 & 1.9058 & 13.3988 & 46.3326 & 3.45796 & 1.2585 & 17.6732 & 97.8013 & 5.53387 & 13.1592 \\
\hline 0.2 & 2.3262 & 21.8879 & 84.3278 & 3.85271 & 2.0316 & 13.7881 & 44.6643 & 3.23935 & 1.3171 & 17.9183 & 96.3202 & 5.37551 & 12.46757 \\
\hline 0.3 & 2.4836 & 22.6347 & 79.7678 & 3.52414 & 2.16725 & 14.2392 & 42.7678 & 3.00353 & 1.3782 & 18.1992 & 94.2929 & 5.18116 & 11.70883 \\
\hline 0.4 & 2.65465 & 23.4938 & 74.9388 & 3.18973 & 2.31419 & 14.7578 & 40.6819 & 2.75664 & 1.44179 & 18.5161 & 91.7608 & 4.95574 & \begin{tabular}{|l|l}
10.90211 \\
\end{tabular} \\
\hline 0.5 & 2.84117 & 24.477 & 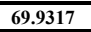 & 2.85704 & 2.4739 & 15.3506 & 38.4482 & 2.50467 & 1.50813 & 18.8694 & 88.7743 & 4.70466 & 10.06637 \\
\hline 0.6 & 3.04522 & 25.5979 & 64.8376 & 2.53293 & 2.64805 & 16.0253 & 36.1102 & 2.25332 & 1.57733 & 19.2599 & 85.3912 & 4.43363 & 9.21988 \\
\hline 0.7 & 3.2691 & 26.8716 & 59.7455 & 2.22337 & 2.8385 & 16.7906 & 33.7125 & 2.00782 & 1.6495 & 19.6881 & 81.6753 & 4.14845 & 8.37964 \\
\hline 0.8 & 3.5151 & 28.3145 & 54.7412 & 1.93333 & 3.047 & 17.656 & 31.2996 & 1.77275 & 1.7249 & 20.155 & 77.6942 & 3.85483 & 7.56091 \\
\hline 0.9 & 3.7859 & 29.9447 & 49.9049 & 1.66657 & 3.2762 & 18.6319 & 28.9153 & 1.55193 & 1.8036 & 20.6613 & 73.5181 & 3.55825 & 6.77675 \\
\hline
\end{tabular}

Table 4: The results of crisp and fuzzy values for negative exponential distribution

\begin{tabular}{|c|c|c|c|c|c|c|c|c|c|c|c|c|c|}
\hline \multirow[b]{2}{*}{$\beta_{r}$} & \multicolumn{4}{|c|}{ Item 1} & \multicolumn{4}{|c|}{ Item 2} & \multicolumn{4}{|c|}{ Item 3} & \multirow[b]{2}{*}{$\operatorname{Min} E(T C)$} \\
\hline & $N_{1}$ & $Q_{m 1}$ & $E(T C)_{1}$ & $\begin{array}{l}E\left(T C_{1}\right)= \\
E(T C) / Q_{m 1}\end{array}$ & $N_{2}$ & $Q_{m 2}$ & $E(T C)_{2}$ & $\begin{array}{l}E\left(T C_{2}\right)= \\
E(T C) / Q_{m 2}\end{array}$ & $N_{3}$ & $Q_{m 3}$ & $E(T C)_{3}$ & $\begin{array}{l}E\left(T C_{3}\right)= \\
E(T C) / Q_{m 3}\end{array}$ & \\
\hline 0.1 & 2.20956 & 21.4862 & 151.975 & 7.07313 & 1.94171 & 13.5711 & 85.0254 & 6.26517 & 1.25852 & $\begin{array}{l}17.7628 \\
\end{array}$ & $\begin{array}{l}174.387 \\
\end{array}$ & 9.81751 & 24.01431 \\
\hline 0.2 & 2.35372 & 22.1267 & 147.837 & 6.6814 & 2.0699 & 13.9729 & 83.8022 & 5.9975 & 1.31598 & 17.9989 & 173.434 & 9.63577 & 23.17625 \\
\hline 0.3 & 2.50934 & 22.8656 & $\begin{array}{l}143.376 \\
\end{array}$ & 6.2704 & 2.20829 & $\begin{array}{l}4.4377 \\
\end{array}$ & 82.4046 & 5.70759 & 1.37575 & 18.2694 & 171.994 & 9.4143 & 22.25665 \\
\hline 0.4 & 2.67792 & 23.712 & $\begin{array}{l}138.68 \\
\end{array}$ & 5.8485 & 2.35823 & 14.9715 & 80.8678 & 5.40144 & 1.43795 & 18.5746 & $\begin{array}{l}170.106 \\
\end{array}$ & 9.15803 & 21.27455 \\
\hline 0.5 & 2.86112 & 24.6763 & 133.833 & 5.42352 & 2.52129 & 15.5811 & 79.2278 & 5.08486 & 1.50271 & $\begin{array}{l}18.9147 \\
\end{array}$ & 167.818 & 8.87235 & 20.24862 \\
\hline 0.6 & 3.06076 & 25.7707 & 128.92 & 5.00259 & 2.69915 & 16.2744 & 77.5206 & 4.76335 & 1.57017 & 19.2902 & 165.182 & 8.56298 & 18.32892 \\
\hline 0.7 & 3.27882 & 27.0081 & \begin{tabular}{l|l|}
124.021 \\
\end{tabular} & 4.592 & 2.89366 & 17.0602 & 75.7805 & 4.44195 & 1.64046 & 19.7018 & 162.258 & 8.23572 & 17.26967 \\
\hline 0.8 & 3.51739 & 28.4029 & 119.211 & 4.19712 & 3.10684 & 17.9482 & 74.0394 & 4.12517 & 1.71372 & 20.15 & 159.111 & 7.89629 & 16.21858 \\
\hline 0.9 & 3.77876 & 29.971 & 114.558 & 3.82228 & 3.34082 & 18.949 & 72.3261 & 3.81687 & 1.79009 & $\begin{array}{l}20.6357 \\
\end{array}$ & $\begin{array}{l}155.803 \\
\end{array}$ & 7.55015 & $\begin{array}{l}15.1893 \\
\end{array}$ \\
\hline$\widetilde{\boldsymbol{\beta}}_{r}$ & $\widetilde{N}_{1}$ & $\widetilde{Q}_{m 1}$ & $E\left(\widetilde{(\widetilde{C})_{1}}\right.$ & $\begin{array}{l}\left.E(\widetilde{T C})_{1}\right) \\
E(\widetilde{T C})_{1} / Q_{n}\end{array}$ & $\widetilde{N}_{2}$ & $\widetilde{Q}_{m 2}$ & $E(\widetilde{T C})_{2}$ & $\begin{array}{l}E E(\widetilde{T C})_{2}= \\
E(\widetilde{T C})_{2} / Q_{m}\end{array}$ & $\widetilde{N}_{3}$ & $\widetilde{Q}_{m 3}$ & $E(\widetilde{T C})_{3}$ & $\begin{array}{l}\left.E(\widetilde{T C})_{3}\right) \\
E(\widetilde{T C})_{3} / Q_{m}\end{array}$ & $\operatorname{Min} E(\widetilde{T C})$ \\
\hline 0.1 & 2.1807 & 21.2434 & 141.1345 & 6.6437 & 1.9058 & 13.3988 & 77.2921 & 5.7686 & 1.2585 & 17.6732 & 164.92 & 9.3316 & 21.7439 \\
\hline 0.2 & 2.3262 & 21.8879 & 137.6016 & 6.2867 & 2.0316 & 13.7881 & 76.2345 & 5.5290 & 1.3171 & 17.9183 & 164.1129 & 9.1589 & 20.9746 \\
\hline 0.3 & 2.4836 & $\begin{array}{l}22.6347 \\
\end{array}$ & 133.7538 & 5.9092 & 2.16725 & 14.2392 & 75.01 & 5.2678 & 1.3782 & 18.1992 & 162.8407 & 8.9477 & 20.1247 \\
\hline 0.4 & 2.65465 & 23.4938 & 129.667 & 5.51923 & 2.31419 & 14.7578 & 73.6475 & 4.99041 & 1.44179 & $\begin{array}{ll}18.5161 \\
\end{array}$ & 161.138 & 8.70262 & 19.21226 \\
\hline 0.5 & 2.84117 & 24.477 & 125.418 & 5.12394 & 2.4739 & 15.3506 & \begin{tabular}{ll|}
72.1831 \\
\end{tabular} & 4.70229 & 1.50813 & 18.8694 & $\begin{array}{l}159.05 \\
\end{array}$ & 8.42898 & 18.25521 \\
\hline 0.6 & 3.04522 & 25.5979 & 121.082 & 4.73014 & 2.64805 & 16.0253 & 70.649 & 4.40859 & 1.57733 & 19.2599 & 156.627 & 8.13228 & 17.27101 \\
\hline 0.7 & 3.2691 & 26.8716 & 116.7282 & 4.3439 & 2.8385 & 16.7906 & 69.077 & 4.114 & 1.6495 & $\begin{array}{l}19.6881 \\
\end{array}$ & 153.9252 & 7.8182 & 16.2761 \\
\hline 0.8 & 3.5151 & 28.3145 & 112.4261 & 3.9706 & 3.047 & 17.656 & 67.4972 & 3.8229 & 1.7249 & 20.155 & 151.0053 & 7.4922 & 15.2857 \\
\hline 0.9 & 3.7859 & 29.9447 & 108.2383 & 3.615 & 3.2762 & 18.6319 & 65.9360 & 3.5389 & 1.8036 & 20.6613 & 147.929 & 7.1597 & \begin{tabular}{ll|}
14.3136 \\
\end{tabular} \\
\hline
\end{tabular}

Table 5: The results of crisp and fuzzy values for exponential distribution

\begin{tabular}{|c|c|c|c|c|c|c|c|c|c|c|c|c|c|}
\hline \multirow[b]{2}{*}{$\boldsymbol{\beta}_{r}$} & \multicolumn{4}{|c|}{ Item 1} & \multicolumn{4}{|c|}{ Item 2} & \multicolumn{4}{|c|}{ Item 3} & \multirow[b]{2}{*}{$\operatorname{Min} E(T C)$} \\
\hline & $N_{1}$ & $Q_{m 1}$ & $E(T C)_{1}$ & $\begin{array}{l}E\left(T C_{1}\right)= \\
E(T C) / Q_{m 1}\end{array}$ & $N_{2}$ & $Q_{m 2}$ & $E(T C)_{2}$ & $\begin{array}{l}E\left(T C_{2}\right)= \\
E(T C) / Q_{m 2}\end{array}$ & $N_{3}$ & $Q_{m 3}$ & $E(T C)_{3}$ & $\begin{array}{l}E\left(T C_{3}\right)= \\
E(T C) / Q_{m 3}\end{array}$ & \\
\hline 0.1 & 2.20956 & 21.4862 & 105.394 & 4.90521 & 1.94171 & 13.5711 & 60.0995 & 4.42848 & 1.25852 & 17.7628 & 117.795 & 6.63156 & 15.96525 \\
\hline 0.2 & 2.35372 & 22.1267 & 100.58 & 4.54567 & 2.0699 & 13.9729 & 58.2424 & 4.16825 & 1.31598 & 17.9989 & 116.186 & 6.45515 & 15.16907 \\
\hline 0.3 & 2.50934 & 22.8656 & $\begin{array}{l}5.4024 \\
\end{array}$ & 4.17231 & 2.20829 & 14.4377 & 56.151 & 3.88919 & 1.37575 & 18.2694 & 114.011 & 6.24051 & \begin{tabular}{|l|l}
14.30201 \\
\end{tabular} \\
\hline 0.4 & 2.67792 & 23.712 & 89.9601 & 3.79386 & 2.35823 & 14.9715 & 53.8678 & 3.59802 & 1.43795 & 18.5746 & 111.314 & 5.99282 & 13.3847 \\
\hline 0.5 & 2.86112 & 24.6763 & 84.3543 & 3.41843 & 2.52129 & 15.5811 & 51.438 & 3.30131 & 1.50271 & 18.9147 & 108.149 & 5.71774 & 12.43748 \\
\hline 0.6 & 3.06076 & 25.7707 & $\begin{array}{l}78.6851 \\
\end{array}$ & $\begin{array}{l}.05328 \\
\end{array}$ & 2.69915 & 16.2744 & 48.9085 & 3.00525 & 1.57017 & 19.2902 & 104.576 & 5.42122 & 11.47975 \\
\hline 0.7 & 3.27882 & 27.0081 & 73.0491 & 2.70471 & 2.89366 & 17.0602 & 46.3272 & 2.71551 & 1.64046 & 19.7018 & 100.663 & 5.10931 & 10.52953 \\
\hline 0.8 & 3.51739 & 28.4029 & 67.5381 & 2.37786 & 3.10684 & 17.9482 & 43.7414 & 2.43709 & 1.71372 & 20.15 & 96.4778 & 4.78797 & 9.60292 \\
\hline 0.9 & 3.77876 & 29.971 & 62.2365 & 2.07656 & 3.34082 & 18.949 & 41.1973 & 2.17411 & 1.79009 & 20.6357 & 92.0945 & 4.46287 & 8.71354 \\
\hline$\widetilde{\boldsymbol{\beta}}_{r}$ & $\widetilde{N}_{1}$ & $\widetilde{Q}_{m 1}$ & $E(\widetilde{T C})_{1}$ & $\begin{array}{l}E(\widetilde{T C})_{1}= \\
E(\widetilde{T C})_{1} / Q_{m 1}\end{array}$ & $\widetilde{N}_{2}$ & $\widetilde{Q}_{m 2}$ & $E(\widetilde{T C})_{2}$ & $\begin{array}{l}E\left(\widetilde{T C} \widetilde{C}_{2}\right)= \\
E(\widetilde{T C})_{2} / Q_{m 2}\end{array}$ & $\widetilde{N}_{3}$ & $\widetilde{Q}_{m 3}$ & $E(\widetilde{T C})_{3}$ & $\begin{array}{l}E\left(\widetilde{T C} C_{3}\right)= \\
E(\widetilde{T C})_{3} / Q_{m 3}\end{array}$ & $\operatorname{Min} E(\widetilde{T C})$ \\
\hline 0.1 & 2.1807 & 21.2434 & 96.9292 & 4.56279 & 1.9058 & 13.3988 & 53.3326 & 3.98039 & 1.2585 & 17.6732 & 109.587 & 6.20074 & 14.74392 \\
\hline 0.2 & 2.3262 & 21.8879 & 92.7278 & 4.23649 & 2.0316 & 13.7881 & 51.6643 & 3.74703 & 1.3171 & 17.9183 & 108.106 & 6.03326 & 14.01678 \\
\hline 0.3 & 2.4836 & 22.6347 & 88.1678 & 3.89525 & 2.16725 & 14.2392 & 49.7678 & 3.49513 & 1.3782 & 18.1992 & 106.079 & 5.82876 & 13.21914 \\
\hline 0.4 & 2.65465 & 23.4938 & 83.3388 & 3.54727 & 2.31419 & 14.7578 & 47.6819 & 3.23096 & 1.44179 & 18.5161 & 103.547 & $5.59225^{\circ}$ & 12.37048 \\
\hline 0.5 & 2.84117 & 24.477 & 78.3317 & 3.20022 & 2.4739 & 15.3506 & 45.4482 & 2.96068 & 1.50813 & 18.8694 & 100.56 & 5.32926 & $\overline{11.49016}$ \\
\hline 0.6 & 3.04522 & 25.5979 & 73.2376 & 2.86108 & 2.64805 & 16.0253 & 43.1102 & 2.69013 & 1.57733 & 19.2599 & 97.177 & 5.04556 & 10.59677 \\
\hline 0.7 & 3.2691 & 26.8716 & 68.1455 & 2.53597 & 2.8385 & 16.7906 & 40.7125 & 2.42472 & 1.6495 & 19.6881 & \begin{tabular}{|c|}
93.461 \\
\end{tabular} & 4.74707 & 9.70776 \\
\hline 0.8 & 3.5151 & 28.3145 & 63.1412 & 2.23 & 3.047 & $\begin{array}{ll}17.656 \\
\end{array}$ & 38.2996 & 2.16922 & 1.7249 & 20.155 & 89.4799 & 4.43959 & 8.83881 \\
\hline 0.9 & 3.7859 & 29.9447 & 58.3049 & 1.94709 & 3.2762 & 18.6319 & 35.9153 & 1.92763 & 1.8036 & 20.6613 & 85.3038 & 4.12867 & 8.00339 \\
\hline
\end{tabular}


Table 6: The results of crisp and fuzzy values for Weibull distribution

\begin{tabular}{|c|c|c|c|c|c|c|c|c|c|c|c|c|c|}
\hline \multirow[b]{2}{*}{$\beta_{r}$} & \multicolumn{4}{|c|}{ Item 1} & \multicolumn{4}{|c|}{ Item 2} & \multicolumn{4}{|c|}{ Item 3} & \multirow[b]{2}{*}{$\operatorname{Min} E(T C)$} \\
\hline & $N_{1}$ & $Q_{m 1}$ & $E(T C)_{1}$ & $\begin{array}{l}E\left(T C_{1}\right)= \\
E(T C) / Q_{m 1}\end{array}$ & $N_{2}$ & $Q_{m 2}$ & $E(T C)_{2}$ & $\begin{array}{l}E\left(T C_{2}\right)= \\
E(T C) / Q_{m 2}\end{array}$ & $N_{3}$ & $Q_{m 3}$ & $E(T C)_{3}$ & $\begin{array}{l}\begin{array}{l}E\left(T C_{3}\right)= \\
E(T C) / Q_{m 3}\end{array}\end{array}$ & \\
\hline 0.1 & 2.20956 & 21.4862 & 105.138 & $\begin{array}{c}4.893291 \\
\end{array}$ & 1.94171 & 13.5711 & 58.5784 & 4.3164 & 1.25852 & 17.7628 & \begin{tabular}{|l|l|}
116.624 \\
\end{tabular} & 6.5656 & 15.77529 \\
\hline 0.2 & 2.35372 & 22.1267 & $\begin{array}{l}100.427 \\
\end{array}$ & 4.53874 & 2.0699 & 13.9729 & 56.8045 & 4.06534 & 1.31598 & 17.9989 & \begin{tabular}{|l|l|}
115.073 \\
\end{tabular} & 6.39331 & 14.99739 \\
\hline 0.3 & 2.50934 & 22.8656 & 955.3639 & 4.17063 & 2.20829 & 14.4377 & 54.8064 & 3.79605 & 1.37575 & 18.2694 & 112.964 & 6.18322 & 14.1499 \\
\hline 0.4 & 2.67792 & 23.712 & 990.0486 & 3.7976 & 2.35823 & 14.9715 & 52.6267 & 3.51512 & 1.43795 & $\begin{array}{l}18.5746 \\
\end{array}$ & \begin{tabular}{|l|l|}
110.341 \\
\end{tabular} & 5.94043 & 13.25315 \\
\hline 0.5 & 2.86112 & 24.6763 & 84.5822 & 3.42766 & 2.52129 & 15.5811 & $\begin{array}{l}50.3108 \\
\end{array}$ & 3.22896 & 1.50271 & 18.9147 & 107.257 & 5.67056 & 12.32718 \\
\hline 0.6 & 3.06076 & 25.7707 & 79.0646 & 3.06801 & 2.69915 & 16.2744 & $\begin{array}{l}47.9055 \\
\end{array}$ & 2.94362 & 1.57017 & 19.2902 & 103.771 & 5.37948 & 11.39111 \\
\hline 0.7 & 3.27882 & $\begin{array}{l}27.0081 \\
\end{array}$ & $\begin{array}{l}73.5926 \\
\end{array}$ & 2.72484 & 2.89366 & 17.0602 & 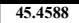 & $\begin{array}{l}2.66441 \\
\end{array}$ & 1.64046 & \begin{tabular}{|l|}
19.7018 \\
\end{tabular} & \begin{tabular}{|l}
99.9513 \\
\end{tabular} & 5.07321 & 10.46266 \\
\hline 0.8 & 3.51739 & 28.4029 & 68.2577 & 2.40319 & 3.10684 & 17.9482 & 43.0179 & 2.39678 & 1.71372 & 20.15 & 95.8666 & 4.75764 & 9.55761 \\
\hline 0.9 & 3.77876 & 29.971 & 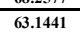 & 2.040684 & 3.34082 & 18.949 & \begin{tabular}{|l|l|}
40.6288 \\
\end{tabular} & 2.14411 & 1.79009 & \begin{tabular}{|l|}
20.6357 \\
\end{tabular} & \begin{tabular}{|l}
9.58002 \\
1.582
\end{tabular} & 4.43838 & 8.68933 \\
\hline$\widetilde{\boldsymbol{\beta}}_{r}$ & $\widetilde{N}_{1}$ & $\widetilde{Q}_{m 1}$ & $E(\widetilde{T C})_{1}$ & $\begin{array}{l}E\left(T \widetilde{T C}_{1}\right)= \\
E(\widetilde{T C}), Q_{0}\end{array}$ & $\tilde{N}_{2}$ & $\tilde{Q}_{m 2}$ & $E(\widetilde{T C})_{2}$ & $\begin{array}{l}\left.E(\widetilde{T C})_{2}\right) \\
E(\widetilde{T C})_{2} / Q_{m}\end{array}$ & $\tilde{N}_{3}$ & $\tilde{Q}_{m 3}$ & $E(\widetilde{T C})_{3}$ & $\begin{array}{l}\left.E(\widetilde{T C})_{3}\right)= \\
E(\widetilde{T C})_{3} / Q_{m}\end{array}$ & $\operatorname{Min} E(\widetilde{T C})$ \\
\hline 0.1 & 2.1807 & 21.2434 & 966.647 & 4.54951 & 1.9058 & 13.3988 & 51.8185 & 3.86738 & 1.2585 & \begin{tabular}{|l}
17.6732 \\
\end{tabular} & \begin{tabular}{|l|l|}
108.414 \\
\end{tabular} & 6.13438 & 14.55127 \\
\hline 0.2 & 2.3262 & 21.8879 & $\begin{array}{l}92.5452 \\
\end{array}$ & 4.22815 & 2.0316 & 13.7881 & 50.2294 & 3.64297 & 1.3171 & 17.9183 & 106.993 & 5.97116 & 13.84228 \\
\hline 0.3 & 2.4836 & 22.6347 & 88.0972 & 3.89213 & 2.16725 & 14.2399 & 48.4222 & 3.40063 & 1.3782 & 18.1992 & 105.034 & 5.77135 & 13.06411 \\
\hline 0.4 & 2.65465 & 23.4938 & 83.3924 & 3.54956 & 2.31419 & 14.7578 & 466.4354 & 3.1465 & 1.44179 & 18.5161 & 102.577 & 5.5399 & 12.23596 \\
\hline 0.5 & 2.84117 & 24 & 78.5222 & 3.208 & 2.4739 & 15.3506 & 444.311 & 2.88659 & 1.50813 & 18.8694 & \begin{tabular}{|l|}
99.6731 \\
\end{tabular} & 5.28225 & 11.37684 \\
\hline 0.6 & 3.04522 & 25.5979 & 73.5774 & 2.87436 & 2.64805 & 16.0253 & 42.0923 & 2.62661 & 1.57733 & 19.2599 & 96.3794 & 5.00415 & 10.50512 \\
\hline 0.7 & 3.2691 & 26.8716 & \begin{tabular}{|c|}
68.6474 \\
\end{tabular} & 2.55465 & 2.8385 & 16.7906 & \begin{tabular}{|l|}
39.824 \\
\end{tabular} & 2.3718 & 1.6495 & 19.6881 & 92.7594 & 4.71144 & 9.63789 \\
\hline 0.8 & 3.5151 & 28.3145 & 63.8177 & 2.25389 & 3.047 & 17.656 & 37.5506 & 2.12679 & 1.7249 & 20.155 & 88.8807 & 4.40986 & 8.79054 \\
\hline 0.9 & 3.7859 & 29.9447 & 59.1682 & 1.97592 & 3.2762 & 18.6319 & 35.3157 & 1.89544 & 1.8036 & 20.6613 & 84.8129 & 4.10491 & 7.97627 \\
\hline
\end{tabular}

Table 7: The results of crisp and fuzzy values for Cauchy distribution

\begin{tabular}{|c|c|c|c|c|c|c|c|c|c|c|c|c|c|}
\hline \multirow[b]{2}{*}{$\boldsymbol{\beta}_{r}$} & \multicolumn{4}{|c|}{ Item 1} & \multicolumn{4}{|c|}{ Item 2} & \multicolumn{4}{|c|}{ Item 3} & \multirow{2}{*}{$\operatorname{Min} E(T C)$} \\
\hline & $N_{1}$ & $Q_{m 1}$ & $E(T C)_{1}$ & $\begin{array}{l}E\left(T C_{1}\right)= \\
E(T C) / Q_{m 1}\end{array}$ & $N_{2}$ & $Q_{m 2}$ & $E(T C)_{2}$ & $\begin{array}{l}E\left(T C_{2}\right)= \\
E(T C) / Q_{m 2}\end{array}$ & $N_{3}$ & $Q_{m 3}$ & $E(T C)_{3}$ & $\begin{array}{l}E\left(T C_{3}\right)= \\
E(T C) / Q_{m 3}\end{array}$ & \\
\hline 0.1 & 2.20956 & 21.4862 & 222.15 & 10.3392 & 1.94171 & 13.5711 & 106.818 & 7.87099 & 1.25852 & 17.7628 & 220.54 & 12.4158 & 30.62599 \\
\hline 0.2 & 2.35372 & 22.1267 & 221.147 & 9.99458 & 2.0699 & 13.9729 & 1008.347 & 7.75408 & 1.31598 & 17.9989 & 221.981 & 12.333 & 30.08166 \\
\hline 0.3 & 2.50934 & 22.8656 & \begin{tabular}{|l}
219.757 \\
\end{tabular} & 9.61084 & 2.20829 & 14.4377 & 1110.083 & 7.6247 & 1.37575 & 18.2694 & 223.263 & 12.2206 & 29.45614 \\
\hline 0.4 & 2.67792 & 23.712 & \begin{tabular}{|l|l|}
217.79 \\
\end{tabular} & 9.18482 & 2.35823 & 14.9715 & 112.058 & 7.48477 & 1.43795 & 18.5746 & 224.412 & 12.0817 & $\begin{array}{l}28.75129 \\
\end{array}$ \\
\hline 0.5 & 2.86112 & 24.6763 & 214.922 & 8.70964 & 2.52129 & 15.5811 & 114.284 & 7.3348 & 1.50271 & 18.9147 & 225.461 & 11.9199 & 27.96434 \\
\hline 0.6 & 3.06076 & 25.7707 & 210.641 & 8.17367 & 915 & 16.2744 & 116.74 & 7.17324 & & 19.2902 & 226.448 & 11.739 & \\
\hline 0.7 & 3.27882 & 27.0081 & 204.167 & 7.55948 & 2.89366 & 17.0602 & 119. & 6.99586 & 446 & 19.7018 & 227.40 & 11.5425 & 99784 \\
\hline \begin{tabular}{l|l}
0.8 \\
\end{tabular} & 3.51739 & 28.4029 & 194.351 & 6.84264 & 3.10684 & 17.9482 & 121.959 & 6.79502 & 1.71372 & 20.15 & 228.373 & 11.3336 & 1.97126 \\
\hline 0.9 & 3.77876 & 29.971 & \begin{tabular}{|l|l|}
179.535 \\
\end{tabular} & 5.99029 & 3.34082 & 18.949 & 124.286 & 6.55893 & 1.79009 & 20.6357 & 229.371 & 11.1152 & 23.66442 \\
\hline$\overline{\widetilde{\beta}_{r}}$ & $\overline{\tilde{N}_{1}}$ & ${\widetilde{\mathbf{Q}_{m 1}}}$ & $E(\widetilde{T C})_{1}$ & $\begin{array}{l}E\left(\overline{T\left(\widetilde{C}_{1}\right)}\right)= \\
F(\bar{T} S)\end{array}$ & $\overline{\tilde{N}_{2}}$ & ${\widetilde{Q_{m 2}}}$ & $E(\widetilde{T C})_{2}$ & $\begin{array}{l}E\left(\overline{T T_{2}}\right)= \\
E \overline{T C})\end{array}$ & $\widetilde{N_{3}}$ & $\widetilde{\tilde{Q}_{m 3}}$ & $E(\widetilde{T C})_{3}$ & $\begin{array}{l}E\left(\widetilde{T C}_{3}\right)= \\
E \overline{T C O})\end{array}$ & $\operatorname{Min} E(\widetilde{T C})$ \\
\hline 0.1 & 2.1807 & 21.2434 & 206.884 & $\begin{array}{r}r\left(1,11 Q_{n}\right. \\
9.73873\end{array}$ & 1.9058 & 13.3988 & 97.3231 & $\frac{1}{7.26354}$ & 1.2585 & 17.6732 & 209.351 & $\frac{1(1.8457}{11.843}$ & 28.84797 \\
\hline 0.2 & 2.3262 & 21.8879 & 206.51 & 9.4349 & 2.0316 & 13.7881 & 98.8707 & 7.17075 & 1.3171 & 17.9183 & 210.993 & 11.7753 & 28.38095 \\
\hline 0.3 & 2.4836 & 22.6347 & \begin{tabular}{|l|l|}
205.81 \\
\end{tabular} & 9.09267 & 2.16725 & & $\begin{array}{l}100.625 \\
\end{array}$ & 7.06675 & 1.37 & 18.1992 & 212.503 & 11.6765 & 27.83592 \\
\hline 0.4 & 2.65465 & $\begin{array}{l}23.4938 \\
\end{array}$ & \begin{tabular}{|l|l|}
204.592 \\
\end{tabular} & 8.70833 & 2.31419 & & $\begin{array}{l}102.617 \\
\end{array}$ & 6.9534 & & 18.5161 & 213.905 & 11.5524 & 27.21413 \\
\hline 0.5 & 2.84117 & 24.477 & 202.531 & & 2.4739 & & 104.864 & & 1.50813 & 18.8694 & 215.232 & 11.4064 & 26.51206 \\
\hline 0.6 & 3.04522 & 25.5979 & \begin{tabular}{|l|l|}
199.108 \\
\end{tabular} & 7.77832 & 2.64805 & 16.0253 & 107.356 & 6.69915 & 1.57733 & 19.2599 & 216.514 & 81.2417 & 25.71917 \\
\hline 0.7 & 3.2691 & 26.8716 & \begin{tabular}{|l|l|}
193.518 \\
\end{tabular} & 7.2016 & 2.8385 & 16.7906 & 110.033 & 6.55327 & 1.6495 & 19.6881 & 217.786 & 11.0618 & 24.81667 \\
\hline 0.8 & 3.5151 & 28.3145 & 184.558 & 6.51815 & 3.047 & 17.656 & 112.765 & 88678 & 1.7249 & 20.155 & 219.075 & 10.8695 & 23.77443 \\
\hline 0.9 & 3.7859 & 29.9447 & \begin{tabular}{|l|l}
170.466 \\
\end{tabular} & 5.69269 & 3.2762 & 18.6319 & 115.312 & 6.18899 & 1.8036 & 20.6613 & 220.402 & 10.6674 & 22.54908 \\
\hline
\end{tabular}

Table 8: The results of crisp and fuzzy values for triangular distribution

\begin{tabular}{|c|c|c|c|c|c|c|c|c|c|c|c|c|c|}
\hline \multirow[b]{2}{*}{$\boldsymbol{\beta}_{r}$} & \multicolumn{4}{|c|}{ Item 1} & \multicolumn{4}{|c|}{ Item 2} & \multicolumn{4}{|c|}{ Item 3} & \multirow[b]{2}{*}{$\operatorname{Min} E(T C)$} \\
\hline & $N_{1}$ & $Q_{m 1}$ & $E(T C)_{1}$ & $\begin{array}{l}E\left(T C_{1}\right)= \\
E(T C) / Q_{m 1}\end{array}$ & $\mathrm{~N}_{2}$ & $Q_{m 2}$ & $E(T C)_{2}$ & $\begin{array}{l}E\left(T C_{2}\right)= \\
E(T C) / Q_{m 2}\end{array}$ & $N_{3}$ & $Q_{m 3}$ & $E(T C)_{3}$ & $\begin{array}{l}E\left(T C_{3}\right)= \\
E(T C) / Q_{m 3}\end{array}$ & \\
\hline 0.1 & 2.20956 & 21.4862 & 149.54 & 6.95981 & 1.94171 & 13.5711 & $\mathbf{8 4 . 9 3 4 3}$ & 6.25846 & 1.25852 & 17.7628 & 211.187 & 11.8893 & 25.10757 \\
\hline 0.2 & 2.35372 & 22.1267 & 144.726 & 6.54081 & 2.0699 & 13.9729 & 83.0793 & 5.94576 & 1.31598 & 17.9989 & 210.147 & 11.6755 & 24.16207 \\
\hline 0.3 & 2.50934 & 22.8656 & 139.548 & 6.10298 & 2.20829 & 14.4377 & 80.9892 & 5.60955 & 1.37575 & 18.2694 & 208.573 & 11.4165 & 23.12903 \\
\hline 0.4 & 2.67792 & 23.712 & 134.106 & 5.65562 & 2.35823 & 14.9715 & 78.7067 & 5.2571 & 1.43795 & 18.5746 & 206.495 & 11.1171 & 22.02982 \\
\hline 0.5 & 2.86112 & 24.6763 & 128.5 & 5.20742 & 2.52129 & 15.5811 & 76.2773 & 4.8955 & 1.50271 & 18.9147 & 203.95 & 10.7826 & 20.88552 \\
\hline 0.6 & 3.06076 & 25.7707 & 122.831 & 4.76631 & 2.69915 & 16.2744 & 73.748 & 4.53154 & 1.57017 & 19.2902 & 200.982 & 10.4189 & 19.71675 \\
\hline 0.7 & 3.27882 & 27.0081 & 117.195 & 4.33925 & 2.89366 & 17.0602 & 71.1666 & 4.1715 & 1.64046 & 19.7018 & 197.646 & 10.0319 & 18.54265 \\
\hline 0.8 & 3.51739 & 28.4029 & 111.684 & 3.93213 & 3.10684 & 17.9482 & 68.5809 & 3.82104 & 1.71372 & 20.15 & 193.999 & 9.62771 & 17.38088 \\
\hline 0.9 & 3.77876 & 29.971 & 106.382 & 3.54951 & 3.34082 & 18.949 & 66.0368 & 3.48497 & 1.79009 & 20.6357 & 190.104 & 9.21236 & 16.24684 \\
\hline$\widetilde{\boldsymbol{\beta}}_{r}$ & $\tilde{N}_{1}$ & $\widetilde{Q}_{m 1}$ & $E(\widetilde{T C})_{1}$ & $\begin{array}{l}E(\widetilde{T C})_{1}= \\
E(\widetilde{T C})_{1} / Q_{m 1}\end{array}$ & $\widetilde{N}_{2}$ & $\tilde{Q}_{m 2}$ & $E(\widetilde{T C})_{2}$ & $\begin{array}{l}E\left(\widetilde{T C}_{2}\right)= \\
E\left(\widetilde{T C}_{2} / Q_{m 2}\right.\end{array}$ & $\widetilde{N}_{3}$ & $\widetilde{Q}_{m 3}$ & $E(\widetilde{T C})_{3}$ & $\begin{array}{l}\left.E(\widetilde{T C})_{3}\right)= \\
E(\widetilde{T C})_{3} / Q_{m 3}\end{array}$ & $\operatorname{Min} E(\widetilde{T C})$ \\
\hline 0.1 & 2.1807 & 21.2434 & 125.909 & 5.92695 & 1.9058 & 13.3988 & 77.4764 & 5.78232 & 1.2585 & 17.6732 & 201.089 & 11.3782 & 23.08747 \\
\hline 0.2 & 2.3262 & 21.8879 & 121.707 & 5.56048 & 2.0316 & 13.7881 & 75.8105 & 5.49828 & 1.3171 & 17.9183 & 200.203 & 11.1731 & 22.23186 \\
\hline 0.3 & 2.4836 & 22.6347 & $\begin{array}{l}117.147 \\
\end{array}$ & 5.17556 & 2.16725 & 14.2392 & 73.9156 & 5.19101 & 1.3782 & 18.1992 & 198.804 & 10.9238 & 21.29037 \\
\hline 0.4 & 2.65465 & 23.4938 & 112.318 & 4.78077 & 2.31419 & 14.7578 & 71.8307 & 4.8673 & 1.44179 & 18.5161 & 196.916 & 10.6349 & 20.28297 \\
\hline 0.5 & 2.84117 & 24.477 & 107.311 & 4.38417 & 2.4739 & 15.3506 & 69.5974 & 4.53385 & 1.50813 & 18.8694 & 194.573 & 10.3115 & 19.22952 \\
\hline 0.6 & 3.04522 & 25.5979 & 102.217 & 3.99318 & 2.64805 & 16.0253 & 67.2596 & 4.19708 & 1.57733 & 19.2599 & 191.816 & 9.95936 & 18.14962 \\
\hline 0.7 & 3.2691 & 26.8716 & 97.1249 & 3.61441 & 2.8385 & 16.7906 & 64.8619 & 3.86299 & 1.6495 & 19.6881 & 188.695 & 9.5842 & 17.0616 \\
\hline 0.8 & 3.5151 & 28.3145 & 92.1206 & 3.25348 & 3.047 & 17.656 & 62.4491 & 3.53699 & 1.7249 & 20.155 & 185.265 & 9.19199 & 15.98246 \\
\hline 0.9 & 3.7859 & 29.9447 & 87.2843 & 2.91485 & 3.2762 & 18.6319 & 60.0648 & 3.22376 & 1.8036 & 20.6613 & 181.586 & 8.78868 & 14.92729 \\
\hline
\end{tabular}


Table 9: The results of crisp and fuzzy values for Rayleigh distribution

\begin{tabular}{|c|c|c|c|c|c|}
\hline $\begin{array}{l}\text { ITEM } 1 \\
--- \text { Crisp E(TC) } \\
- \text { Fuzzy E(TC) }\end{array}$ & 50 & $\begin{array}{l}\text { ITEM } 2 \\
\qquad \begin{array}{r}--\operatorname{Crisp} E(T C) \\
\quad \text { Fuzzy } E(T C)\end{array}\end{array}$ & 5 읍 & $\begin{array}{l}\text { ITEM } 3 \\
\begin{array}{c}-- \text { Crisp E(TC) } \\
- \text { Fuzzy E(TC) }\end{array}\end{array}$ & 10 \\
\hline $\begin{array}{c}0.5 \\
\boldsymbol{\beta}\end{array}$ & 0 & $\stackrel{0.5}{\boldsymbol{\beta}}$ & 0 & $\begin{array}{c}0.5 \\
\boldsymbol{\beta}\end{array}$ & 0 \\
\hline
\end{tabular}

Figure 3: Crisp and fuzzy value for uniform distribution

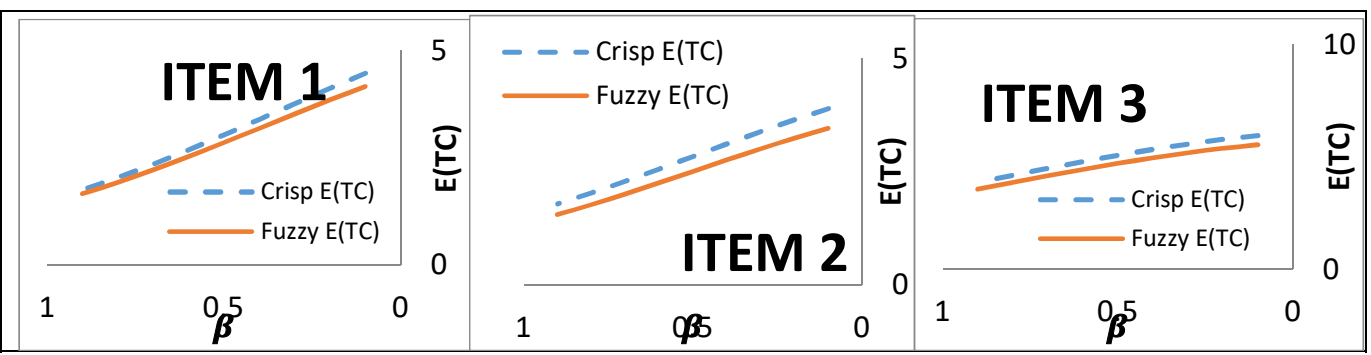

Figure 4: Crisp and fuzzy value for negative exponential

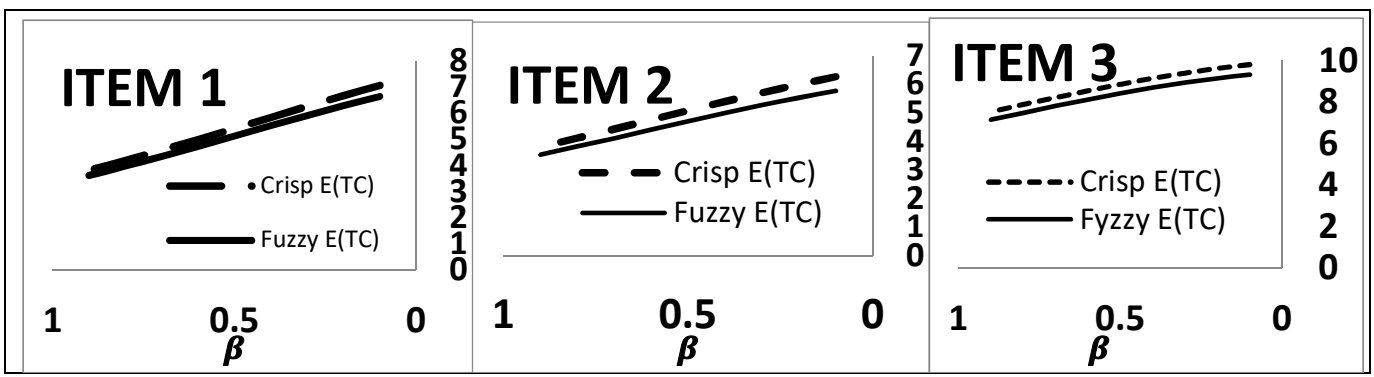

Figure 5: Crisp and fuzzy value for exponential distribution

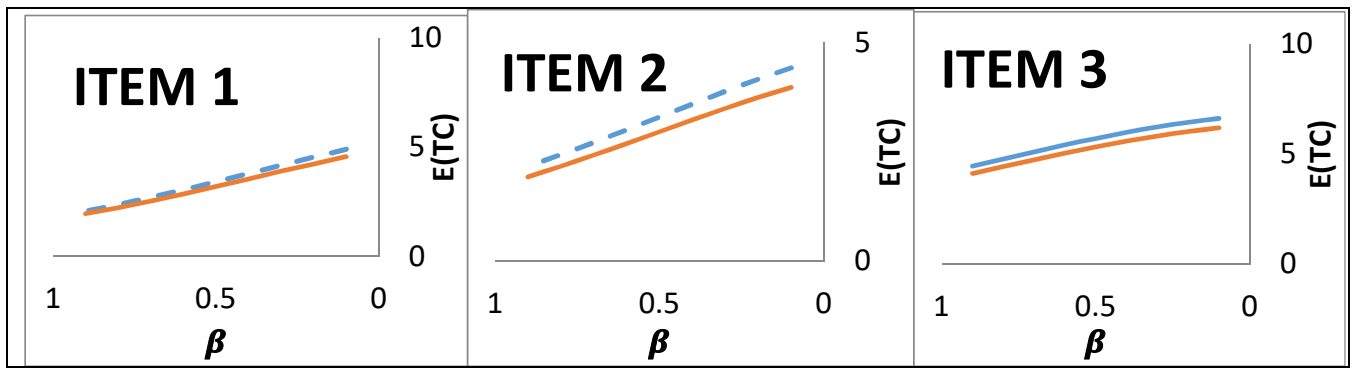

Figure 6: Crisp and fuzzy value for Weibull distribution

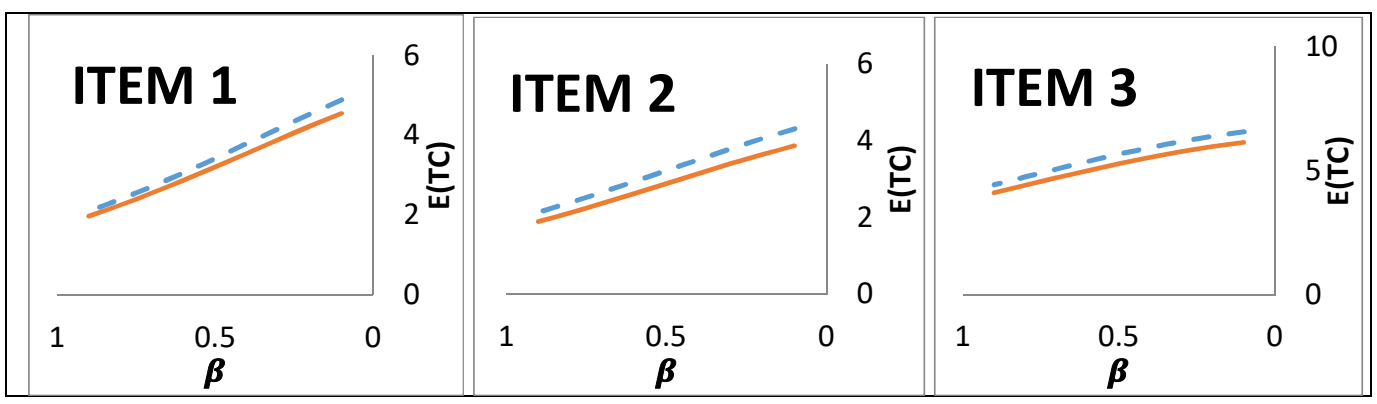


Figure 7: Crisp and fuzzy value for Cauchy distribution

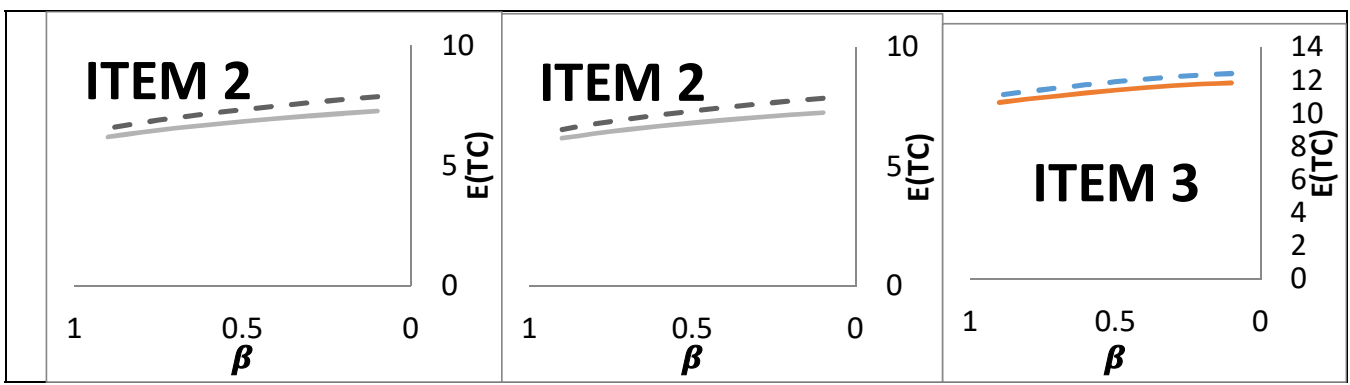

Figure 8: Crisp and fuzzy value for triangular distribution

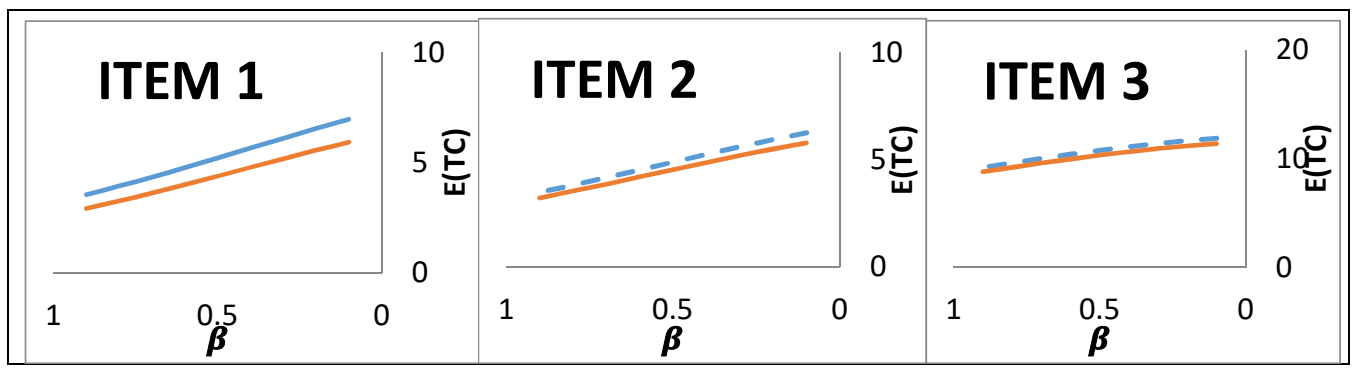

Figure 9: Crisp and fuzzy value for Rayleigh distribution

\section{Conclusion}

In this paper, we developed a probabilistic Multi-item single-source (MISS) inventory model with varying holding cost under a restriction, which is on storage space. We determine the optimal review period and optimal maximum inventory level that minimized the expected annual total cost under constraint using Geometric programming technique for crisp and TFN environment. When $\beta$ convergence to 1 , the solution approaching to the optimal solution.

\section{References}

C. Chiang, Periodic Review Inventory Models with Stochastic Supplier's Visit Intervals, International Journal of Production Economics, 115 (2008) 433-438.

E. A. Silver, D. F. Pyke, R. Peterson, Inventory Management and Production Planning and Scheduling, John Wiley and Sons, New York, 1998.

Farshid Samadi, Abolfazl Mirzazadeh, Mir Mohsen Pedram, Fuzzy Pricing, Marketing and Service Planning In a Fuzzy Inventory Model: A Geometric Programming Approach, Applied

S. Boyd, S .J. Kim, L Vandenberghe, A. Hassibi, a Tutorial on Geometric Programming, Optim. Eng., 8 (2007) 67-127.
Mathematical Modelling Journal, 137 (2013) 6683-6694.

Fergany. H. A., Periodic Review Probabilistic Multi-Item Inventory System with Zero Lead Time under Constraints and Varying Ordering Cost. American Journal of Applied Sciences, 2(8) (2005), 1213-1217.

K. A. M. Kotb, Fergany H. A., Multi-Item EOQ Model with Varying Holding Cost: A Geometric Programming Approach, International Mathematical Forum, Vol. 6, (2011), no. 23, 1135 1144

M. J. Soble, R. Q. Zhang, Inventory Polices For Systems with Stochastic and Deterministic Demand, Operations Research, 49 (2001) 157162.

M.O. Abou-El-Ata, K.A.M. Kotb, MultiItem EOQ Inventory Model with Varying Holding Cost under Two Restrictions: A Geometric Programming Approach, Production Planning \& Control 8 (5) (1997) 608-611.

M.O. Abuo-El-Ata, Hala A. Fergany, Mona F. El-Wakeel, Probabilistic Multi-Item Inventory Model With Varying Order Cost under Two Restrictions: A Geometric Programming Approach, Int. J. Production Economics, 83 (2003) 223-231.

R. J. Duffin, E.L. Peterson, C. Zener, Geometric Programming Theory and Applications, Wiley, New York, 1966.

S. Islam, Multi-Objective Marketing Planning Inventory Model: A Geometric 
Programming Approach, Appl. Math, Computer, 205 (2008) 238-246.

S. Islam, T.K. Roy, Fuzzy Multi-Item Economic Production Quantity Model under Space Constraint: A Geometric Programming Approach, Appl. Math. Computer, 184 (2007) 326-335.

S.A. Tarim, B.G. Kingsman, Modeling And Computing $\left(R^{n}, S^{n}\right)$ Policies For Inventory Systems with Non-Stationary Stochastic Demand, European Journal of Operational Research , 174 (2006) 581-599

S.A. Tarim, B.M. Smith, Constraint Programming For Computing Non-Stationary $(\mathrm{R}, \mathrm{S})$ Inventory Policies, European Journal of Operational Research, 189 (2008) 1004-1021

S.J. Sadjadi, M. Ghazanfari, A. Yousefli, Fuzzy Pricing and Marketing Planning Model: A
Possibilistic Geometric Programming Approach, Expert Syst. Appl. 37 (2010) 3392-3397.

S.T. Liu, Geometric Programming with Fuzzy Parameters In Engineering Optimization, Int. J. Approx. Reason., 46 (2007) 484-498.

Shashank Grag, D. Krishna Sundar, K. Ravikumar, A Periodic Tabular Policy For Scheduling of A Single Stage Production-Inventory System, Computers \& Industrial Engineering, 62 (2012) 21-28.

T. Iida, The Infinite Horizon NonStationary Stochastic Inventory Problem: Near Myopic Polices And Weak Ergodicity, European Journal of Operational Research. 116 (1999) 405-422.

Y. Feng, B. Xiao, A New Algorithm For Computing Optimal $(\mathrm{s}, \mathrm{S})$ Policies In A Stochastic Single Item/Location Inventory System, IIE Transactions, 32 (2000) 1081-1090.

يعرف المخزون السلعي علي أنه تخزين الخامات وتجهيز ها لمواجهة الطلب. ولقد حدثت مشكلة التخزين عندما كان من الضروري

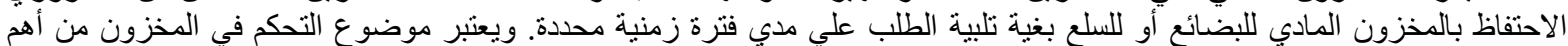

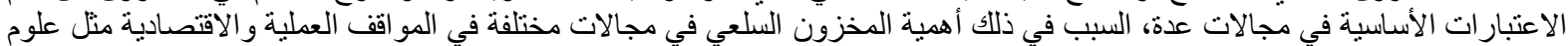

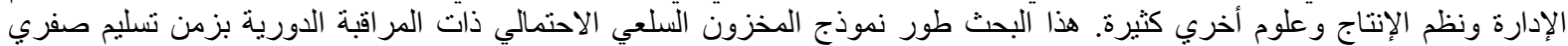

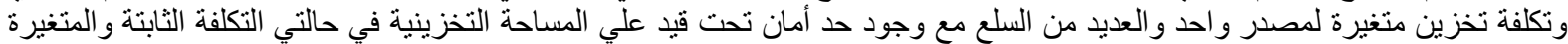

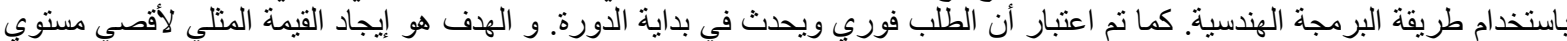
للمخزون وزمن المر اقبة الأمثل الذي يعطي أقل تكلفة إجمالية سنوية. ثم اتبع بمثال عددي لتوفي لتوضيح النتائج. 